\title{
Effects of sfermion mixing induced by RGE running in the minimal flavor violating CMSSM
}

\author{
M. E. Gómez ${ }^{1, \mathrm{a}}$, S. Heinemeyer ${ }^{2, \mathrm{~b}}$, M. Rehman ${ }^{2, \mathrm{c}}$ \\ ${ }^{1}$ Department of Applied Physics, University of Huelva, 21071 Huelva, Spain \\ ${ }^{2}$ Instituto de Física de Cantabria (CSIC-UC), 39005 Santander, Spain
}

Received: 15 January 2015 / Accepted: 2 September 2015 / Published online: 21 September 2015

(C) The Author(s) 2015. This article is published with open access at Springerlink.com

\begin{abstract}
Within the Constrained Minimal Supersymmetric Standard Model (CMSSM) with Minimal Flavor Violation (MFV) for scalar quarks, we study numerically the effects of intergenerational squark mixing on $B$-physics observables, electroweak precision observables (EWPO), and the Higgs-boson mass predictions. In models with universal soft terms at the GUT scale, squark mixing is generated through the Renormalization Group Equations (RGEs) running from the GUT scale to the electroweak scale due to presence of non-diagonal Yukawa matrices in the RGEs, e.g. due to the CKM matrix. Our numerical analysis is based on the code Spheno for the RGE running and full one-loop calculations, supplemented by further higher-order corrections, at the electroweak scale of the precision observables as included in the code FeynHiggs. Taking the CMSSM as a concrete "realistic" example, we find that the $B$-physics observables as well as the Higgs mass predictions do not receive sizable corrections. On the other hand, in our numerical analysis we observe that the EWPO such as the $W$ boson mass can receive relevant corrections. Such contributions could in principle be used to place new bounds on the CMSSM parameter space. We extend our numerical analysis to the CMSSM extended with a mechanism to explain neutrino masses (CMSSMseesaw I), which induces flavor violation in the scalar lepton sector. The effects of slepton mixing on the analyzed observables are found to be, in general, smaller than those of squark mixing, but in our numerical analysis reach the level of the current experimental uncertainty for the EWPO.
\end{abstract}

M. Rehman is a MulitDark Scholar.

\footnotetext{
a e-mail: mario.gomez@dfa.uhu.es

b e-mail: Sven.Heinemeyer@cern.ch

c e-mail: rehman@ifca.unican.es
}

\section{Introduction}

Supersymmetric (SUSY) extensions of the Standard Model (SM) are broadly considered as the most motivated and promising New Physics (NP) theories beyond the SM. The solution of the hierarchy problem, the gauge coupling unification and the possibility of having a natural cold dark matter candidate, constitute the most convincing arguments in favor of SUSY.

Within the Minimal Supersymmetric Standard Model (MSSM) [1-3], flavor mixing can occur in both scalar quark and scalar lepton sector. Here the possible presence of soft SUSY-breaking parameters in the squark and slepton sector, which are off-diagonal in flavor space (mass parameters as well as trilinear couplings) are the most general way to introduce flavor mixing within the MSSM. This, however, yields many new sources of flavor and $\mathcal{C P}$-violation, which potentially lead to large non-standard effects in flavor processes, in conflict with the experimental bounds.

The SM has been very successfully tested by low-energy flavor observables both from the kaon and the $B_{d}$ sectors. In particular, the two $B$ factories have established that $B_{d}$ flavor and $\mathcal{C P}$-violating processes are well described by the SM up to an accuracy of the $\sim 10 \%$ level [4]. This immediately implies a tension between the solution of the hierarchy problem, calling for a NP scale at or below the TeV scale, and the explanation of the flavor physics data, requiring a multi-TeV NP scale, if the new flavor-violating couplings are of generic size.

An elegant way to simultaneously solve the above problems is provided by the Minimal Flavor Violation (MFV) hypothesis [5-8], where flavor and $\mathcal{C P}$-violation in the quark sector are assumed to be entirely described by the CKM matrix, even in theories beyond the SM. For example in MSSM, the off-diagonality in the sfermion mass matrix reflects the misalignment (in flavor space) between fermions and sfermions mass matrices, which cannot be diagonalized 
simultaneously. This misalignment can be produced from various origins. For instance, off-diagonal sfermion mass matrix entries can be generated by Renormalization Group Equations (RGE) running. Going from a high energy scale, where no flavor violation is assumed, down to the electroweak (EW) scale, such entries can be generated due to the presence of non-diagonal Yukawa matrices in the RGEs. For instance, in the Constrained Minimal Supersymmetric Standard Model (CMSSM, see [9] and references therein), the RGE effects on non-diagonal sfermion soft SUSY-breaking parameters are affected only by non-diagonal elements on the Yukawa couplings and the trilinear terms which are taken as proportional to the Yukawas at the GUT scale. We choose the following form of the Yukawa matrices (working in the Super-CKM basis [10]):

$$
Y_{D}=\operatorname{diag}\left(y_{d}, y_{s}, y_{b}\right), \quad Y_{U}=V_{\mathrm{CKM}}^{\dagger} \operatorname{diag}\left(y_{u}, y_{c}, y_{t}\right) .
$$

Hence, all flavor violation in the quark and squark sector is generated by the RGEs and controlled by the CKM matrix, i.e. the Yukawa couplings have a strong impact on the size of the induced off-diagonal entries in the squark mass matrices.

The situation is somewhat different in the slepton sector where neutrinos are strictly massless (in the SM and the MSSM). Consequently, there is no slepton mixing, which would induce Lepton Flavor Violation (LFV) in the charged sector, allowing not yet observed processes like $l_{i} \rightarrow l_{j} \gamma$ $\left(i>j ; l_{3,2,1}=\tau, \mu, e\right)$ [11]. However, in the neutral sector, we have strong experimental evidence that shows that the neutrinos are massive and mix among themselves [1220]. In order to incorporate this, one needs to go beyond the MSSM to introduce a mechanism that generates neutrino masses. The simplest way would be to introduce Dirac masses, leaving, however, the extreme smallness of the neutrino masses unexplained. To overcome this problem, typically a seesaw mechanism is used to generate neutrino masses, and the PMNS matrix plays the role of the CKM matrix in the lepton sector. Extending the MFV hypothesis for leptons [21] we can assume that the flavor mixing in the lepton and slepton sector is induced and controlled by the seesaw mechanism.

Consequently, in this paper we will numerically investigate two "realistic" models (more detailed definitions are given in the next section):

(i) the CMSSM, where only flavor violation in the squark sector is present;

(ii) the CMSSM augmented by the seesaw type I mechanism [22-28], called "CMSSM-seesaw I" below.

In many analyses of the CMSSM, or extensions such as the NUHM1 or NUHM2 (see [9] and references therein), the hypothesis of MFV has been used, and it has been assumed that the contributions coming from MFV are negligible for other observables as well; see, e.g., [29-32]. In this paper we will perform a numerical analysis to see whether this assumption is justified, and whether including these MFV effects could in principle lead to additional constraints on the CMSSM parameter space. However, we do not attempt to find analytical solutions to analyze this, as they become extremely involved in the presence of intergenerational mixing in SUSY models. In this respect we numerically evaluate in the CMSSM and in the CMSSM-seesaw I the following set of observables: $B$ physics observables (BPO), in particular $\operatorname{BR}\left(B \rightarrow X_{s} \gamma\right), \operatorname{BR}\left(B_{s} \rightarrow \mu^{+} \mu^{-}\right)$and $\Delta M_{B_{s}}$, electroweak precision observables (EWPO), in particular $M_{W}$ and the effective weak leptonic mixing angle, $\sin ^{2} \theta_{\text {eff }}$, as well as the masses of the neutral and charged Higgs bosons in the MSSM.

In order to perform our calculations, we use the code SPheno [33,34] to generate the CMSSM (containing also the type I seesaw) particle spectrum by running RGE from the GUT down to the EW scale. The effects of the CKM matrix in the RGE running on the mixing in the scalar fermion sector thus fully relies on the SPheno implementation. The particle spectrum was then handed over in the form of an SLHA file $[10,35]$ to FeynHiggs [36-41] to calculate EWPO and Higgs-boson masses. The $B$ physics observables were calculated by the BPHYSICS subroutine included in the SuFla code $[42,43]$ (see also [44-46] for the improved version used here).

Our setup provides an evaluation of flavor-violating effects in "realistic" MFV models (where flavor violation enters only via RGE running) using state-of-the-art tools, compared to state-of-the-art limits, where the size of the effects will also be compared to future sensitivities. Effects that may appear negligible now might be non-negligible in the future. Furthermore, in the case of lepton-flavor violation [case (ii) above], we are not aware of any analysis of this type.

The paper is organized as follows: First we review the main features of the MSSM with sfermion flavor mixing in MFV in Sect. 2. The computational setup is given in Sect. 3. The numerical results are presented in Sect. 4, where first we discuss the effect of squarks mixing in the CMSSM. In a second step we analyze the effects of slepton mixing i.e. the CMSSM-seesaw I. Our conclusions can be found in Sect. 5.

\section{Model setup}

In this section we will first review the CMSSM and the concept of MFV. Subsequently, we will discuss the MSSM, its seesaw extension and parameterization of sfermion mixing at low energy. 


\subsection{The CMSSM and MFV}

The MSSM is the simplest supersymmetric structure we can build from the SM particle content. The general setup for the soft SUSY-breaking parameters is given by [1-3]

$$
\begin{aligned}
-\mathcal{L}_{\text {soft }}= & \left(m_{\tilde{Q}}^{2}\right)_{i}^{j} \tilde{q}_{L}^{\dagger i} \tilde{q}_{L j}+\left(m_{\tilde{u}}^{2}\right)_{j}^{i} \tilde{u}_{R i}^{*} \tilde{u}_{R}^{j}+\left(m_{\tilde{d}}^{2}\right)_{j}^{i} \tilde{d}_{R i}^{*} \tilde{d}_{R}^{j} \\
& +\left(m_{\tilde{L}}^{2}\right)_{i}^{j} \tilde{l}_{L}^{\dagger} \tilde{l}_{L j}+\left(m_{\tilde{e}}^{2}\right)_{j}^{i} \tilde{e}_{R i}^{*} \tilde{e}_{R}^{j}+\tilde{m}_{1}^{2} h_{1}^{\dagger} h_{1} \\
& +\tilde{m}_{2}^{2} h_{2}^{\dagger} h_{2}+\left(B \mu h_{1} h_{2}+\text { h.c. }\right)+\left(A_{d}^{i j} h_{1} \tilde{d}_{R i}^{*} \tilde{q}_{L j}\right. \\
& +A_{u}^{i j} h_{2} \tilde{u}_{R i}^{*} \tilde{q}_{L j}+A_{l}^{i j} h_{1} \tilde{e}_{R i}^{*} \tilde{l}_{L j}+\frac{1}{2} M_{1} \tilde{B}_{L}^{0} \tilde{B}_{L}^{0} \\
& \left.+\frac{1}{2} M_{2} \tilde{W}_{L}^{a} \tilde{W}_{L}^{a}+\frac{1}{2} M_{3} \tilde{G}^{a} \tilde{G}^{a}+\text { h.c. }\right) .
\end{aligned}
$$

Here $m_{\tilde{Q}}^{2}$ and $m_{\tilde{L}}^{2}$ are $3 \times 3$ matrices in family space (with $i, j$ being the generation indices) for the soft masses of the left-handed squark $\tilde{q}_{L}$ and slepton $\tilde{l}_{L} S U(2)$ doublets, respectively. $m_{\tilde{u}}^{2}, m_{\tilde{d}}^{2}$, and $m_{\tilde{e}}^{2}$ contain the soft masses for right-handed up-type squarks $\tilde{u}_{R}$, down-type squarks $\tilde{d}_{R}$, and charged slepton $\tilde{e}_{R} S U(2)$ singlets, respectively. $A_{u}, A_{d}$, and $A_{l}$ are the $3 \times 3$ matrices for the trilinear couplings for up-type squarks, down-type squarks, and charged slepton, respectively; $\tilde{m}_{1}$ and $\tilde{m}_{2}$ are the soft masses of the Higgs sector. In the last line $M_{1}, M_{2}$, and $M_{3}$ define the bino, wino, and gluino mass terms, respectively.

Within the constrained MSSM the soft SUSY-breaking parameters are assumed to be universal at the Grand Unification scale $M_{\mathrm{GUT}} \sim 2 \times 10^{16} \mathrm{GeV}$,

$$
\begin{aligned}
& \left(m_{Q}^{2}\right)_{i j}=\left(m_{U}^{2}\right)_{i j}=\left(m_{D}^{2}\right)_{i j}=\left(m_{L}^{2}\right)_{i j}=\left(m_{E}^{2}\right)_{i j}=m_{0}^{2} \delta_{i j}, \\
& m_{H_{1}}^{2}=m_{H_{2}}^{2}=m_{0}^{2}, \quad m_{\tilde{g}}=m_{\tilde{W}}=m_{\tilde{B}}=m_{1 / 2} \\
& \left(A_{U}\right)_{i j}=A_{0} e^{i \phi_{A}}\left(Y_{U}\right)_{i j}, \quad\left(A_{D}\right)_{i j}=A_{0} e^{i \phi_{A}}\left(Y_{D}\right)_{i j} \\
& \left(A_{E}\right)_{i j}=A_{0} e^{i \phi_{A}}\left(Y_{E}\right)_{i j} .
\end{aligned}
$$

There is a common mass for all the scalars, $m_{0}^{2}$, a single gaugino mass, $m_{1 / 2}$, and all the trilinear soft-breaking terms are directly proportional to the corresponding Yukawa couplings in the superpotential with a proportionality constant $A_{0} e^{i \phi_{A}}$, containing a potential non-trivial complex phase.

With the use of the Renormalization Group Equations (RGE) of the MSSM, one can obtain the SUSY spectrum at the EW scale. All the SUSY masses and mixings are then given as a function of $m_{0}^{2}, m_{1 / 2}, A_{0}$, and $\tan \beta=v_{2} / v_{1}$, the ratio of the two vacuum expectation values (see below). We require radiative symmetry breaking to fix $|\mu|$ and $|B \mu|$ $[47,48]$ with the tree-level Higgs potential.

By definition, this model fulfills the MFV hypothesis, since the only flavor-violating terms stem from the CKM matrix. The important point is that, even in a model with universal soft SUSY-breaking terms at some high energy scale as the CMSSM, some off-diagonality in the squark mass matrices appears at the EW scale. Working in the basis where the squarks are rotated parallel to the quarks, the so-called Super CKM (SCKM) basis, the squark mass matrices are not flavor diagonal at the EW scale. This is due to the fact that at $M_{\mathrm{GUT}}$ there exist two non-trivial flavor structures, namely the two Yukawa matrices for the up and down quarks, which are not simultaneously diagonalizable. This implies that through RGE evolution some flavor mixing leaks into the sfermion mass matrices. In a general SUSY model the presence of new flavor structures in the soft SUSY-breaking terms would generate large flavor mixing in the sfermion mass matrices. However, in the CMSSM, which we are investigating here, the two Yukawa matrices are the only source of flavor change. As always in the SCKM basis, any off-diagonal entry in the sfermion mass matrices at the EW scale will be necessarily proportional to a product of Yukawa couplings. The RGEs for the soft SUSY-breaking terms are sets of linear equations, and, thus, to match the correct chirality of the coupling, Yukawa couplings or trilinear soft terms must enter the RGE in pairs. (The same holds for the CMSSM-seesaw I; see below.)

\subsection{MSSM and its seesaw extension}

One can write the most general $S U(3)_{C} \times S U(2)_{L} \times U(1)_{Y}$ gauge invariant and renormalizable superpotential as

$$
\begin{aligned}
W_{\mathrm{MSSM}}= & Y_{e}^{i j} \epsilon_{\alpha \beta} H_{1}^{\alpha} E_{i}^{c} L_{j}^{\beta}+Y_{d}^{i j} \epsilon_{\alpha \beta} H_{1}^{\alpha} D_{i}^{c} Q_{j}^{\beta} \\
& +Y_{u}^{i j} \epsilon_{\alpha \beta} H_{2}^{\alpha} U_{i}^{c} Q_{j}^{\beta}+\mu \epsilon_{\alpha \beta} H_{1}^{\alpha} H_{2}^{\beta}
\end{aligned}
$$

where $L_{i}$ represents the chiral multiplet of a $S U(2)_{L}$ doublet lepton, $E_{i}^{c}$ a $S U(2)_{L}$ singlet charged lepton, $H_{1}$ and $H_{2}$ two Higgs doublets with opposite hypercharge. Similarly $Q, U$, and $D$ represent chiral multiplets of quarks of a $S U(2)_{L}$ doublet and two singlets with different $U(1)_{Y}$ charges. Three generations of leptons and quarks are assumed and thus the subscripts $i$ and $j$ run over 1 to 3 . The symbol $\epsilon_{\alpha \beta}$ is an anti-symmetric tensor with $\epsilon_{12}=1$.

In order to provide an explanation for the (small) neutrino masses, the MSSM can be extended by the type-I seesaw mechanism [22-28]. The superpotential for CMSSMseesaw I can be written as

$$
W=W_{\mathrm{MSSM}}+Y_{\nu}^{i j} \epsilon_{\alpha \beta} H_{2}^{\alpha} N_{i}^{c} L_{j}^{\beta}+\frac{1}{2} M_{N}^{i j} N_{i}^{c} N_{j}^{c},
$$

where $W_{\text {MSSM }}$ is given in Eq. (4) and $N_{i}^{c}$ is the additional superfield that contains the three right-handed neutrinos, $v_{R i}$, and their scalar partners, $\tilde{v}_{R i} . M_{N}^{i j}$ denotes the $3 \times 3$ Majorana mass matrix for heavy right-handed neutrino. The full set of soft SUSY-breaking terms is given by 


$$
\begin{aligned}
-\mathcal{L}_{\text {soft }, \text { SI }}= & -\mathcal{L}_{\text {soft }}+\left(m_{\tilde{v}}^{2}\right)_{j}^{i} \tilde{v}_{R i}^{*} \tilde{v}_{R}^{j} \\
& +\left(\frac{1}{2} B_{v}^{i j} M_{N}^{i j} \tilde{v}_{R i}^{*} \tilde{v}_{R j}^{*}+A_{v}^{i j} h_{2} \tilde{v}_{R i}^{*} \tilde{l}_{L j}+\text { h.c. }\right),
\end{aligned}
$$

with $\mathcal{L}_{\text {soft }}$ given by Eq. (2), $\left(m_{\tilde{v}}^{2}\right)_{j}^{i}, A_{v}^{i j}$, and $B_{v}^{i j}$ are the new soft-breaking parameters.

By the seesaw mechanism three of the neutral fields acquire heavy masses and decouple at high energy scale that we will denote $M_{N}$; below this scale the effective theory contains the MSSM plus an operator that provides masses to the neutrinos.

$W=W_{\mathrm{MSSM}}+\frac{1}{2}\left(Y_{v} L H_{2}\right)^{T} M_{N}^{-1}\left(Y_{v} L H_{2}\right)$.

This framework naturally explains neutrino oscillations in agreement with experimental data [12-20]. At the electroweak scale an effective Majorana mass matrix for light neutrinos,

$m_{\mathrm{eff}}=-\frac{1}{2} v_{u}^{2} Y_{v} \cdot M_{N}^{-1} \cdot Y_{v}^{T}$,

arises from Dirac neutrino Yukawa $Y_{v}$ (which can be assumed of the same order as the charged-lepton and quark Yukawas), and heavy Majorana masses $M_{N}$. The smallness of the neutrino masses implies that the scale $M_{N}$ is very high, $\mathcal{O}\left(10^{14} \mathrm{GeV}\right)$.

From Eqs. (5) and (6) we can observe that one can choose a basis such that the Yukawa coupling matrix, $Y_{l}^{i j}$, and the mass matrix of the right-handed neutrinos, $M_{N}^{i j}$, are diagonalized as $Y_{l}^{\delta}$ and $M_{R}^{\delta}$, respectively. In this case the neutrino Yukawa couplings $Y_{v}^{i j}$ are not generally diagonal, giving rise to LFV. Here it is important to note that the lepton-flavor conservation is not a consequence of the SM gauge symmetry, even in the absence of the righthanded neutrinos. Consequently, slepton-mass terms can violate the lepton-flavor conservation in a manner consistent with the gauge symmetry. Thus the scale of LFV can be identified with the EW scale, much lower than the righthanded neutrino scale $M_{N}$, leading to potentially observable rates.

In the SM augmented by right-handed neutrinos, the flavor-violating processes such as $\mu \rightarrow e \gamma, \tau \rightarrow \mu \gamma$ etc., whose rates are proportional to inverse powers of $M_{R}^{\delta}$, would be highly suppressed with such a large $M_{N}$ scale, and hence are far beyond current experimental bounds. However, in SUSY theories, the neutrino Dirac couplings $Y_{\nu}$ enter in the RGEs of the soft SUSY-breaking sneutrino and slepton masses, generating LFV. In the basis where the chargedlepton masses $Y_{\ell}$ is diagonal, the soft slepton-mass matrix acquires corrections that contain off-diagonal contributions from the RGE running from $M_{\mathrm{GUT}}$ down to the Majorana mass scale $M_{N}$, of the following form (in the leading-log approximation) [49]:

$$
\begin{aligned}
& \left(m_{\tilde{L}}^{2}\right)_{i j} \sim \frac{1}{16 \pi^{2}}\left(6 m_{0}^{2}+2 A_{0}^{2}\right)\left(Y_{\nu}^{\dagger} Y_{\nu}\right)_{i j} \log \left(\frac{M_{\mathrm{GUT}}}{M_{N}}\right) \\
& \left(m_{\tilde{e}}^{2}\right)_{i j} \sim 0 \\
& \left(A_{l}\right)_{i j} \sim \frac{3}{8 \pi^{2}} A_{0} Y_{l i}\left(Y_{\nu}^{\dagger}{ }^{\dagger} Y_{\nu}\right)_{i j} \log \left(\frac{M_{\mathrm{GUT}}}{M_{N}}\right)
\end{aligned}
$$

Consequently, even if the soft scalar masses were universal at the unification scale, quantum corrections between the GUT scale and the seesaw scale $M_{N}$ would modify this structure via renormalization-group running, which generates offdiagonal contributions [50-55] at $M_{N}$ in a basis such that $Y_{\ell}$ is diagonal. Below this scale, the off-diagonal contributions remain almost unchanged.

Therefore the seesaw mechanism induces non-trivial values for slepton $\delta_{i j}^{F A B}$ resulting in a prediction for LFV decays $l_{i} \rightarrow l_{j} \gamma,(i>j)$ that can be much larger than the nonSUSY case. These rates depend on the structure of $Y_{v}$ at a seesaw scale $M_{N}$ in a basis where $Y_{l}$ and $M_{N}$ are diagonal. By using the approach of [55] a general form of $Y_{v}$ containing all neutrino experimental information can be written as

$Y_{\nu}=\frac{\sqrt{2}}{v_{u}} \sqrt{M_{R}^{\delta}} R \sqrt{m_{\nu}^{\delta}} U^{\dagger}$,

where $R$ is a general orthogonal matrix and $m_{v}^{\delta}$ denotes the diagonalized neutrino mass matrix. In this basis the matrix $U$ can be identified with the $U_{\text {PMNS }}$ matrix obtained as

$m_{\nu}^{\delta}=U^{T} m_{\mathrm{eff}} U$

In order to find values for the slepton generation mixing parameters we need a specific form of the product $Y_{\nu}^{\dagger} Y_{v}$ as shown in Eq. (9). The simple consideration of direct hierarchical neutrinos with a common scale for right-handed neutrinos provides a representative reference value. In this case using Eq. (10) we find

$Y_{\nu}^{\dagger} Y_{\nu}=\frac{2}{v_{u}^{2}} M_{R} U m_{\nu}^{\delta} U^{\dagger}$

Here $M_{R}$ is the common mass assigned to the $v_{R}$. In the conditions considered here, LFV effects are independent of the matrix $R$.

For the numerical analysis the values of the Yukawa couplings etc. have to be set to yield values in agreement with the experimental data for neutrino masses and mixings. In our computation, by considering a normal hierarchy among the neutrino masses, we fix $m_{\nu_{3}} \sim \sqrt{\Delta m_{\mathrm{atm}}^{2}} \sim 0.05 \mathrm{eV}$ and require $m_{v_{2}} / m_{v_{3}}=0.17, m_{v_{2}} \sim 100 \cdot m_{v_{1}}$ consistent with the measured values of $\Delta m_{\mathrm{sol}}^{2}$ and $\Delta m_{\mathrm{atm}}^{2}$ [56]. The matrix $U$ 
is identified with $U_{\text {PMNS }}$ with the $\mathcal{C P}$-phases set to zero and neutrino mixing angles set to the center of their experimental values.

One can observe that $m_{\text {eff }}$ remains unchanged by consistent changes on the scales of $M_{N}$ and $Y_{v}$. This is no longer correct for the off-diagonal entries in the slepton-mass matrices (parameterized by slepton $\delta_{i j}^{F A B}$, see the next subsection). These quantities have a quadratic dependence on $Y_{v}$ and a logarithmic dependence on $M_{N}$; see Eq. (9). Therefore larger values of $M_{N}$ imply larger LFV effects. By setting $M_{N}=10^{14} \mathrm{GeV}$, the largest values of $Y_{\nu}$ are of about 0.29 , this implies an important restriction on the parameters space arising from the $\mathrm{BR}(\mu \rightarrow e \gamma)$ as will be discussed in Sects. 3 and 4. An example of models with almost degenerate $v_{R}$ can be found in [50]. For our numerical analysis we tested several scenarios and we found that the one defined here is the simplest and also the one with larger LFV prediction.

\subsection{Scalar fermion sector with flavor mixing}

In this section we give a brief description about how we parameterize flavor mixing at the EW scale. We are using the same notation as in $[44-46,57,58]$. However, while in this section we give a general description, in our analysis below, contrary to our previous analyses [57], this time we concentrate on the origin of the flavor mixing as discussed in the previous sections.

The most general hypothesis for flavor mixing assumes a mass matrix that is not diagonal in flavor space, both for squarks and sleptons. In the squarks sector and charged slepton sector we have $6 \times 6$ mass matrices, based on the corresponding six electroweak interaction eigenstates, $\tilde{U}_{L, R}$ with $U=u, c, t$ for up-type squarks, $\tilde{D}_{L, R}$ with $D=d, s, b$ for down-type squarks and $\tilde{L}_{L, R}$ with $L=e, \mu, \tau$ for charged sleptons. For the sneutrinos we have a $3 \times 3$ mass matrix, since within the MSSM even with type I seesaw (right-handed neutrinos decouple below their respective mass scale) we have only three electroweak interaction eigenstates, $\tilde{v}_{L}$ with $v=v_{e}, v_{\mu}, v_{\tau}$.

The non-diagonal entries in this $6 \times 6$ general matrix for sfermions can be described in terms of a set of dimensionless parameters $\delta_{i j}^{F A B}(F=Q, U, D, L, E ; A, B=L, R ; i, j=$ $1,2,3, i \neq j$ ) where $F$ identifies the sfermion type, $L, R$ refer to the "left-" and "right-handed" SUSY partners of the corresponding fermionic degrees of freedom, and $i, j$ indices run over the three generations. (Non-zero values for the $\delta_{i j}^{F A B}$ are generated via the processes discussed in the previous subsections.)

One usually writes the $6 \times 6$ non-diagonal mass matrices, $\mathcal{M}_{\tilde{u}}^{2}$ and $\mathcal{M}_{\tilde{d}}^{2}$, referred to the Super-CKM basis, being ordered, respectively, as $\left(\tilde{u}_{L}, \tilde{c}_{L}, \tilde{t}_{L}, \tilde{u}_{R}, \tilde{c}_{R}, \tilde{t}_{R}\right),\left(\tilde{d}_{L}, \tilde{s}_{L}\right.$, $\left.\tilde{b}_{L}, \tilde{d}_{R}, \tilde{s}_{R}, \tilde{b}_{R}\right)$ and $\mathcal{M}_{\tilde{l}}^{2}$ referred to the Super-PMNS basis, being ordered as $\left(\tilde{e}_{L}, \tilde{\mu}_{L}, \tilde{\tau}_{L}, \tilde{e}_{R}, \tilde{\mu}_{R}, \tilde{\tau}_{R}\right)$, and write them in terms of left- and right-handed blocks $M_{\tilde{q} A B}^{2}, M_{\tilde{l} A B}^{2}$ $(q=u, d, A, B=L, R)$, which are non-diagonal $3 \times 3$ matrices,

$\mathcal{M}_{\tilde{q}}^{2}=\left(\begin{array}{cc}M_{\tilde{q} L L}^{2} & M_{\tilde{q} L R}^{2} \\ M_{\tilde{q} L R}^{2 \dagger} & M_{\tilde{q} R R}^{2}\end{array}\right), \quad \tilde{q}=\tilde{u}, \tilde{d}$,

where

$$
\begin{aligned}
M_{\tilde{u} L L i j}^{2} & =m_{\tilde{U}_{L} i j}^{2}+\left(m_{u_{i}}^{2}+\left(T_{3}^{u}-Q_{u} s_{\mathrm{w}}^{2}\right) M_{Z}^{2} \cos 2 \beta\right) \delta_{i j}, \\
M_{\tilde{u} R R i j}^{2} & =m_{\tilde{U}_{R} i j}^{2}+\left(m_{u_{i}}^{2}+Q_{u} s_{\mathrm{w}}^{2} M_{Z}^{2} \cos 2 \beta\right) \delta_{i j}, \\
M_{\tilde{u} L R i j}^{2} & =\left\langle\mathcal{H}_{2}^{0}\right\rangle \mathcal{A}_{i j}^{u}-m_{u_{i}} \mu \cot \beta \delta_{i j}, \\
M_{\tilde{d} L L i j}^{2} & =m_{\tilde{D}_{L} i j}^{2}+\left(m_{d_{i}}^{2}+\left(T_{3}^{d}-Q_{d} s_{\mathrm{w}}^{2}\right) M_{Z}^{2} \cos 2 \beta\right) \delta_{i j}, \\
M_{\tilde{d} R R i j}^{2} & =m_{\tilde{D}_{R} i j}^{2}+\left(m_{d_{i}}^{2}+Q_{d} s_{\mathrm{w}}^{2} M_{Z}^{2} \cos 2 \beta\right) \delta_{i j}, \\
M_{\tilde{d} L R i j}^{2} & =\left\langle\mathcal{H}_{1}^{0}\right\rangle \mathcal{A}_{i j}^{d}-m_{d_{i}} \mu \tan \beta \delta_{i j},
\end{aligned}
$$

and

$$
\mathcal{M}_{\tilde{l}}^{2}=\left(\begin{array}{ll}
M_{\tilde{l} L L}^{2} & M_{\tilde{l} L R}^{2} \\
M_{\tilde{l} L R}^{2 \dagger} & M_{\tilde{l} R R}^{2}
\end{array}\right),
$$

where

$$
\begin{aligned}
& M_{\tilde{l} L L i j}^{2}=m_{\tilde{L} i j}^{2}+\left(m_{l_{i}}^{2}+\left(-\frac{1}{2}+s_{\mathrm{w}}^{2}\right) M_{Z}^{2} \cos 2 \beta\right) \delta_{i j} \\
& M_{\tilde{l} R R i j}^{2}=m_{\tilde{E} i j}^{2}+\left(m_{l_{i}}^{2}-s_{\mathrm{w}}^{2} M_{Z}^{2} \cos 2 \beta\right) \delta_{i j} \\
& M_{\tilde{l} L R i j}^{2}=\left\langle\mathcal{H}_{1}^{0}\right\rangle \mathcal{A}_{i j}^{l}-m_{l_{i}} \mu \tan \beta \delta_{i j}
\end{aligned}
$$

with, $i, j=1,2,3, Q_{u}=2 / 3, Q_{d}=-1 / 3, T_{3}^{u}=1 / 2$, and $T_{3}^{d}=-1 / 2$. The $M_{Z, W}$ denote the $Z$ and $W$ boson masses, with $s_{\mathrm{w}}^{2}=1-M_{W}^{2} / M_{Z}^{2}=1-c_{\mathrm{w}}^{2}$, and $\left(m_{u_{1}}, m_{u_{2}}, m_{u_{3}}\right)=$ $\left(m_{u}, m_{c}, m_{t}\right),\left(m_{d_{1}}, m_{d_{2}}, m_{d_{3}}\right)=\left(m_{d}, m_{s}, m_{b}\right)$ are the quark masses and $\left(m_{l_{1}}, m_{l_{2}}, m_{l_{3}}\right)=\left(m_{e}, m_{\mu}, m_{\tau}\right)$ are the lepton masses. $\mu$ is the Higgsino mass term and $\tan \beta=$ $v_{2} / v_{1}$ with $v_{1}=\left\langle\mathcal{H}_{1}^{0}\right\rangle$ and $v_{2}=\left\langle\mathcal{H}_{2}^{0}\right\rangle$ being the two vacuum expectation values of the corresponding neutral Higgs boson in the Higgs $S U(2)_{L}$ doublets, $\mathcal{H}_{1}=\left(\begin{array}{ll}\mathcal{H}_{1}^{0} & \mathcal{H}_{1}^{-}\end{array}\right)$and $\mathcal{H}_{2}=\left(\mathcal{H}_{2}^{+} \mathcal{H}_{2}^{0}\right)$.

It should be noted that the non-diagonality in flavor comes exclusively from the soft SUSY-breaking parameters, that could be non-vanishing for $i \neq j$, namely: the masses $m_{\tilde{Q}_{i j}}$ and $m_{\tilde{L}_{i j}}$ for the sfermion $S U(2)$ doublets, the masses $m_{\tilde{U}_{L} i j}^{2}, m_{\tilde{U}_{R} i j}^{2}, m_{\tilde{D}_{L} i j}^{2}, m_{\tilde{D}_{R} i j}^{2}, m_{\tilde{E}_{i j}}$ for the sfermion $S U(2)$ singlets and the trilinear couplings, $\mathcal{A}_{i j}^{f}$.

In the sneutrino sector there is, correspondingly, a oneblock $3 \times 3$ mass matrix, that is referred to the $\left(\tilde{v}_{e L}, \tilde{v}_{\mu L}, \tilde{v}_{\tau L}\right)$ electroweak interaction basis: 
$\mathcal{M}_{\tilde{v}}^{2}=\left(M_{\tilde{v} L L}^{2}\right)$,

where

$M_{\tilde{v} L L i j}^{2}=m_{\tilde{L} i j}^{2}+\left(\frac{1}{2} M_{Z}^{2} \cos 2 \beta\right) \delta_{i j}$.

It is important to note that due to $S U(2)_{L}$ gauge invariance the same soft masses $m_{\tilde{Q}_{i j}}$ enter in both up-type and down-type squarks mass matrices similarly $m_{\tilde{L} i j}$ enter in both the slepton and the sneutrino $L L$ mass matrices. The soft SUSY-breaking parameters for the up-type squarks differ from corresponding ones for down-type squarks by a rotation with CKM matrix. The same would hold for sleptons i.e. the soft SUSY-breaking parameters of the sneutrinos would differ from the corresponding ones for charged sleptons by a rotation with the PMNS matrix. However, taking the neutrino masses and oscillations into account in the SM leads to LFV effects that are extremely small. For instance, in $\mu \rightarrow e \gamma$ they are of $\mathcal{O}\left(10^{-47}\right)$ in the case of Dirac neutrinos with mass around $1 \mathrm{eV}$ and maximal mixing [59-62], and of $\mathcal{O}\left(10^{-40}\right)$ in the case of Majorana neutrinos $[59,62]$. Consequently we do not expect large effects from the inclusion of neutrino mass effects here and neglect a rotation with the PMNS matrix. The sfermion mass matrices in terms of the $\delta_{i j}^{F A B}$ are given as

$m_{\tilde{U}_{L}}^{2}=\left(\begin{array}{ccc}m_{\tilde{Q}_{1}}^{2} & \delta_{12}^{Q L L} m_{\tilde{Q}_{1}} m_{\tilde{Q}_{2}} & \delta_{13}^{Q L L} m_{\tilde{Q}_{1}} m_{\tilde{Q}_{3}} \\ \delta_{21}^{Q L L} m_{\tilde{Q}_{2}} m_{\tilde{Q}_{1}} & m_{\tilde{Q}_{2}}^{2} & \delta_{23}^{Q L L} m_{\tilde{Q}_{2}} m_{\tilde{Q}_{3}} \\ \delta_{31}^{Q L L} m_{\tilde{Q}_{3}} m_{\tilde{Q}_{1}} & \delta_{32}^{Q L L} m_{\tilde{Q}_{3}} m_{\tilde{Q}_{2}} & m_{\tilde{Q}_{3}}^{2}\end{array}\right)$,

$m_{\tilde{D}_{L}}^{2}=V_{\mathrm{CKM}}^{\dagger} m_{\tilde{U}_{L}}^{2} V_{\mathrm{CKM}}$,

$m_{\tilde{U}_{R}}^{2}=\left(\begin{array}{ccc}m_{\tilde{U}_{1}}^{2} & \delta_{12}^{U R R} m_{\tilde{U}_{1}} m_{\tilde{U}_{2}} & \delta_{13}^{U R R} m_{\tilde{U}_{1}} m_{\tilde{U}_{3}} \\ \delta_{21}^{U R R} m_{\tilde{U}_{2}} m_{\tilde{U}_{1}} & m_{\tilde{U}_{2}}^{2} & \delta_{23}^{U R R} m_{\tilde{U}_{2}} m_{\tilde{U}_{3}} \\ \delta_{31}^{U R R} m_{\tilde{U}_{3}} m_{\tilde{U}_{1}} & \delta_{32}^{U R R} m_{\tilde{U}_{3}} m_{\tilde{U}_{2}} & m_{\tilde{U}_{3}}^{2}\end{array}\right)$,

$m_{\tilde{D}_{R}}^{2}=\left(\begin{array}{ccc}m_{\tilde{D}_{1}}^{2} & \delta_{12}^{D R R} m_{\tilde{D}_{1}} m_{\tilde{D}_{2}} & \delta_{13}^{D R R} m_{\tilde{D}_{1}} m_{\tilde{D}_{3}} \\ \delta_{21}^{D R R} m_{\tilde{D}_{2}} m_{\tilde{D}_{1}} & m_{\tilde{D}_{2}}^{2} & \delta_{23}^{D R R} m_{\tilde{D}_{2}} m_{\tilde{D}_{3}} \\ \delta_{31}^{D R R} m_{\tilde{D}_{3}} m_{\tilde{D}_{1}} & \delta_{32}^{D R R} m_{\tilde{D}_{3}} m_{\tilde{D}_{2}} & m_{\tilde{D}_{3}}^{2}\end{array}\right)$,

$v_{2} \mathcal{A}^{u}=\left(\begin{array}{ccc}m_{u} A_{u} & \delta_{12}^{U L R} m_{\tilde{Q}_{1}} m_{\tilde{U}_{2}} & \delta_{13}^{U L R} m_{\tilde{Q}_{1}} m_{\tilde{U}_{3}} \\ \delta_{21}^{U L R} m_{\tilde{Q}_{2}} m_{\tilde{U}_{1}} & m_{c} A_{c} & \delta_{23}^{U L R} m_{\tilde{Q}_{2}} m_{\tilde{U}_{3}} \\ \delta_{31}^{U L R} m_{\tilde{Q}_{3}} m_{\tilde{U}_{1}} & \delta_{32}^{U L R} m_{\tilde{Q}_{3}} m_{\tilde{U}_{2}} & m_{t} A_{t}\end{array}\right)$, $v_{1} \mathcal{A}^{d}=\left(\begin{array}{ccc}m_{d} A_{d} & \delta_{12}^{D L R} m_{\tilde{Q}_{1}} m_{\tilde{D}_{2}} & \delta_{13}^{D L R} m_{\tilde{Q}_{1}} m_{\tilde{D}_{3}} \\ \delta_{21}^{D L R} m_{\tilde{Q}_{2}} m_{\tilde{D}_{1}} & m_{s} A_{s} & \delta_{23}^{D L R} m_{\tilde{Q}_{2}} m_{\tilde{D}_{3}} \\ \delta_{31}^{D L R} m_{\tilde{Q}_{3}} m_{\tilde{D}_{1}} & \delta_{32}^{D L R} m_{\tilde{Q}_{3}} m_{\tilde{D}_{2}} & m_{b} A_{b}\end{array}\right)$,

$m_{\tilde{L}}^{2}=\left(\begin{array}{ccc}m_{\tilde{L}_{1}}^{2} & \delta_{12}^{L L L} m_{\tilde{L}_{1}} m_{\tilde{L}_{2}} & \delta_{13}^{L L L} m_{\tilde{L}_{1}} m_{\tilde{L}_{3}} \\ \delta_{21}^{L L L} m_{\tilde{L}_{2}} m_{\tilde{L}_{1}} & m_{\tilde{L}_{2}}^{2} & \delta_{23}^{L L L} m_{\tilde{L}_{2}} m_{\tilde{L}_{3}} \\ \delta_{31}^{L L L} m_{\tilde{L}_{3}} m_{\tilde{L}_{1}} & \delta_{32}^{L L L} m_{\tilde{L}_{3}} m_{\tilde{L}_{2}} & m_{\tilde{L}_{3}}^{2}\end{array}\right)$,

$v_{1} \mathcal{A}^{l}=\left(\begin{array}{ccc}m_{e} A_{e} & \delta_{12}^{E L R} m_{\tilde{L}_{1}} m_{\tilde{E}_{2}} & \delta_{13}^{E L R} m_{\tilde{L}_{1}} m_{\tilde{E}_{3}} \\ \delta_{21}^{E L R} m_{\tilde{L}_{2}} m_{\tilde{E}_{1}} & m_{\mu} A_{\mu} & \delta_{23}^{E L R} m_{\tilde{L}_{2}} m_{\tilde{E}_{3}} \\ \delta_{31}^{E L R} m_{\tilde{L}_{3}} m_{\tilde{E}_{1}} & \delta_{32}^{E L R} m_{\tilde{L}_{3}} m_{\tilde{E}_{2}} & m_{\tau} A_{\tau}\end{array}\right)$,

$m_{\tilde{E}}^{2}=\left(\begin{array}{ccc}m_{\tilde{E}_{1}}^{2} & \delta_{12}^{E R R} m_{\tilde{E}_{1}} m_{\tilde{E}_{2}} & \delta_{13}^{E R R} m_{\tilde{E}_{1}} m_{\tilde{E}_{3}} \\ \delta_{21}^{E R R} m_{\tilde{E}_{2}} m_{\tilde{E}_{1}} & m_{\tilde{E}_{2}}^{2} & \delta_{23}^{E R R} m_{\tilde{E}_{2}} m_{\tilde{E}_{3}} \\ \delta_{31}^{E R R} m_{\tilde{E}_{3}} m_{\tilde{E}_{1}} & \delta_{32}^{E R R} m_{\tilde{E}_{3}} m_{\tilde{E}_{2}} & m_{\tilde{E}_{3}}^{2}\end{array}\right)$.

In all this work, for simplicity, we are assuming that all $\delta_{i j}^{F A B}$ parameters are real and, therefore, hermiticity of $\mathcal{M}_{\tilde{Q}}^{2}$, $\mathcal{M}_{\tilde{l}}^{2}$, and $\mathcal{M}_{\tilde{v}}^{2}$ implies $\delta_{i j}^{F A B}=\delta_{j i}^{F B A}$.

The next step is to rotate the squark states from the SuperCKM basis, $\tilde{q}_{L, R}$, to the physical basis. If we set the order in the Super-CKM basis as above, $\left(\tilde{u}_{L}, \tilde{c}_{L}, \tilde{t}_{L}, \tilde{u}_{R}, \tilde{c}_{R}, \tilde{t}_{R}\right)$ and $\left(\tilde{d}_{L}, \tilde{s}_{L}, \tilde{b}_{L}, \tilde{d}_{R}, \tilde{s}_{R}, \tilde{b}_{R}\right)$, and in the physical basis as $\tilde{u}_{1, \ldots, 6}$ and $\tilde{d}_{1, \ldots, 6}$, respectively, these last rotations are given by two $6 \times 6$ matrices, $R^{\tilde{u}}$ and $R^{\tilde{d}}$,

$\left(\begin{array}{c}\tilde{u}_{1} \\ \tilde{u}_{2} \\ \tilde{u}_{3} \\ \tilde{u}_{4} \\ \tilde{u}_{5} \\ \tilde{u}_{6}\end{array}\right)=R^{\tilde{u}}\left(\begin{array}{c}\tilde{u}_{L} \\ \tilde{c}_{L} \\ \tilde{t}_{L} \\ \tilde{u}_{R} \\ \tilde{c}_{R} \\ \tilde{t}_{R}\end{array}\right), \quad\left(\begin{array}{c}\tilde{d}_{1} \\ \tilde{d}_{2} \\ \tilde{d}_{3} \\ \tilde{d}_{4} \\ \tilde{d}_{5} \\ \tilde{d}_{6}\end{array}\right)=R^{\tilde{d}}\left(\begin{array}{c}\tilde{d}_{L} \\ \tilde{s}_{L} \\ \tilde{b}_{L} \\ \tilde{d}_{R} \\ \tilde{s}_{R} \\ \tilde{b}_{R}\end{array}\right)$,

yielding the diagonal mass-squared matrices for squarks as follows:

$$
\begin{gathered}
\operatorname{diag}\left\{m_{\tilde{u}_{1}}^{2}, m_{\tilde{u}_{2}}^{2}, m_{\tilde{u}_{3}}^{2}, m_{\tilde{u}_{4}}^{2}, m_{\tilde{u}_{5}}^{2}, m_{\tilde{u}_{6}}^{2}\right\}=R^{\tilde{u}} \mathcal{M}_{\tilde{u}}^{2} R^{\tilde{u} \dagger}, \\
\operatorname{diag}\left\{m_{\tilde{d}_{1}}^{2}, m_{\tilde{d}_{2}}^{2}, m_{\tilde{d}_{3}}^{2}, m_{\tilde{d}_{4}}^{2}, m_{\tilde{d}_{5}}^{2}, m_{\tilde{d}_{6}}^{2}\right\}=R^{\tilde{d}} \mathcal{M}_{\tilde{d}}^{2} R^{\tilde{d} \dagger} .
\end{gathered}
$$

Similarly we need to rotate the sleptons and sneutrinos from the electroweak interaction basis to the physical mass eigenstate basis, 


$$
\left(\begin{array}{l}
\tilde{l}_{1} \\
\tilde{l}_{2} \\
\tilde{l}_{3} \\
\tilde{l}_{4} \\
\tilde{l}_{5} \\
\tilde{l}_{6}
\end{array}\right)=R^{\tilde{l}}\left(\begin{array}{c}
\tilde{e}_{L} \\
\tilde{\mu}_{L} \\
\tilde{\tau}_{L} \\
\tilde{e}_{R} \\
\tilde{\mu}_{R} \\
\tilde{\tau}_{R}
\end{array}\right), \quad\left(\begin{array}{c}
\tilde{v}_{1} \\
\tilde{v}_{2} \\
\tilde{v}_{3}
\end{array}\right)=R^{\tilde{v}}\left(\begin{array}{c}
\tilde{v}_{e L} \\
\tilde{v}_{\mu L} \\
\tilde{v}_{\tau L}
\end{array}\right),
$$

with $R^{\tilde{l}}$ and $R^{\tilde{v}}$ being the respective $6 \times 6$ and $3 \times 3$ unitary rotating matrices that yield the diagonal mass-squared matrices as follows:

$$
\begin{aligned}
\operatorname{diag}\left\{m_{\tilde{l}_{1}}^{2}, m_{\tilde{l}_{2}}^{2}, m_{\tilde{l}_{3}}^{2}, m_{\tilde{l}_{4}}^{2}, m_{\tilde{l}_{5}}^{2}, m_{\tilde{l}_{6}}^{2}\right\} & =R^{\tilde{l}} \mathcal{M}_{\tilde{l}}^{2} R^{\tilde{l} \dagger}, \\
\operatorname{diag}\left\{m_{\tilde{\nu}_{1}}^{2}, m_{\tilde{\nu}_{2}}^{2}, m_{\tilde{\nu}_{3}}^{2}\right\} & =R^{\tilde{\nu}} \mathcal{M}_{\tilde{\nu}}^{2} R^{\tilde{\nu} \dagger} .
\end{aligned}
$$

\section{Computational setup}

Here we briefly describe our numerical setup. We first give some details on the running from the GUT to the EW scale, and subsequently describe the calculations of the observables evaluated at the EW scale.

\subsection{From the GUT scale to the EW scale}

The SUSY spectra have been generated with the code SPheno 3.2.4 [33,34] (for the CMSSM and the CMSSMseesaw I). We defined the SLHA $[10,35]$ file at the GUT scale. In a first step within SPheno, gauge and Yukawa couplings at $M_{Z}$ scale are calculated using tree-level formulas. Fermion masses, the $Z$ boson pole mass, the fine-structure constant $\alpha$, the Fermi constant $G_{F}$, and the strong coupling constant $\alpha_{s}\left(M_{Z}\right)$ are used as input parameters. The gauge and Yukawa couplings, calculated at $M_{Z}$, are then used as input for the one-loop RGEs to obtain the corresponding values at the GUT scale which is calculated from the requirement that $g_{1}=g_{2}$ (where $g_{1,2}$ denote the gauge couplings of the $U(1)$ and $S U$ (2), respectively). The CMSSM boundary conditions are then applied to the complete set of two-loop RGEs and are evolved to the EW scale. At this point the SM and SUSY radiative corrections are applied to the gauge and Yukawa couplings, and the two-loop RGEs are again evolved to GUT scale. After applying the CMSSM boundary conditions again the two-loop RGEs are run down to EW scale to get SUSY spectrum. This procedure is iterated until the required precision is achieved. As stressed above, for the effects of the CKM matrix on the sfermion mixing we fully rely on Spheno. The output is then written in the form of an SLHA file, which is used as input to calculate low-energy observables discussed below.

For the CMSSM-seesaw I a similar procedure is applied, where the neutrino related input parameters are included in the respective SLHA input blocks (see [10,35] for details), the relevant numerical values are given in Sect. 2.2. For our scans of the CMSSM-seesaw I parameter space we use SPheno 3.2.4 [33,34] with the model "seesaw type-I". The value for $Y_{v}$ is implemented as explained in Sect. 2.2, adjusting the matrix elements such that neutrino experimental parameters achieve the desired results after RGEs. The predictions for $\mathrm{BR}\left(l_{i} \rightarrow l_{j} \gamma\right)$ are also obtained with SPheno 3.2.4, see the discussion in Sect. 4.2. We checked that the use of this code produces results similar to the ones obtained by our private codes used in [50].

\subsection{Calculations at the EW scale}

Here we briefly review the various observables that we compute at the EW scale, either taking the non-zero $\delta_{i j}^{F A B}$ into account, or setting them to zero.

\subsubsection{The MSSM Higgs sector}

The MSSM Higgs sector consist of two Higgs doublets and predicts five physical Higgs bosons, the light and heavy $\mathcal{C P}$-even $h$ and $H$, the $\mathcal{C P}$-odd $A$, and the charged Higgs boson, $H^{ \pm}$. At tree level the Higgs sector is described with the help of two parameters: the mass of the $A$ boson, $M_{A}$, and $\tan \beta=v_{2} / v_{1}$, the ratio of the two vacuum expectation values. The tree-level relations receive large higher-order corrections; see, e.g., [63,64] and references therein.

The lightest MSSM Higgs boson, with mass $M_{h}$, can be interpreted as the new state discovered at the LHC around $125 \mathrm{GeV}$. The present experimental uncertainty at the LHC for $M_{h}$, is about $[65,66]$,

$\delta M_{h}^{\text {exp,today }} \sim 200 \mathrm{MeV}$.

This can possibly be reduced below the level of

$\delta M_{h}^{\text {exp, future }} \lesssim 50 \mathrm{MeV}$

at the ILC [67]. Similarly, for the masses of the heavy neutral Higgs $M_{H}$ and charged Higgs boson $M_{H^{ \pm}}$, an uncertainty at the $1 \%$ level could be expected at the LHC [68].

Effects of sfermion mixing in the MSSM Higgs sector has already been calculated in a model independent way in the scalar quark sector [44-46,69], as well as independently in [70]. They have also been calculated in the scalar lepton sector in [57]. In both cases there are sizable corrections to the Higgs-boson masses, specially to the charged Higgsboson mass $M_{H^{ \pm}}$, assuming general NMFV in the squark and slepton sector.

In the Feynman diagrammatic approach that we are following here, the higher-order corrected $\mathcal{C P}$-even Higgs- 
boson masses are derived by finding the poles of the $(h, H)$ propagator matrix. The inverse of this matrix is given by

$$
\begin{aligned}
& \left(\Delta_{\text {Higgs }}\right)^{-1} \\
& \quad=-i\left(\begin{array}{ll}
p^{2}-m_{H, \text { tree }}^{2}+\hat{\Sigma}_{H H}\left(p^{2}\right) & \hat{\Sigma}_{h H}\left(p^{2}\right) \\
\hat{\Sigma}_{h H}\left(p^{2}\right) & p^{2}-m_{h, \text { tree }}^{2}+\hat{\Sigma}_{h h}\left(p^{2}\right)
\end{array}\right) .
\end{aligned}
$$

Determining the poles of the matrix $\Delta_{\text {Higgs }}$ in Eq. (36) is equivalent to solving the equation

$$
\begin{aligned}
& {\left[p^{2}-m_{h, \text { tree }}^{2}+\hat{\Sigma}_{h h}\left(p^{2}\right)\right]\left[p^{2}-m_{H, \text { tree }}^{2}+\hat{\Sigma}_{H H}\left(p^{2}\right)\right]} \\
& -\left[\hat{\Sigma}_{h H}\left(p^{2}\right)\right]^{2}=0 .
\end{aligned}
$$

Similarly, in the case of the charged Higgs sector, the corrected Higgs mass is derived by the position of the pole in the charged Higgs propagator, which is defined by

$$
p^{2}-m_{H^{ \pm}, \text {tree }}^{2}+\hat{\Sigma}_{H^{-} H^{+}}\left(p^{2}\right)=0 .
$$

The flavor-violating parameters enter into the one-loop prediction of the various (renormalized) Higgs-boson selfenergies, where details can be found in [44-46,57]. Numerically the results have been obtained using the code FeynHiggs [36-41], which contains the complete set of one-loop corrections from (flavor-violating) squark and slepton contributions (based on $[44,45,57,69])$. Those are supplemented with leading and sub-leading two-loop corrections as well as a resummation of leading and sub-leading logarithmic contributions from the $t / \tilde{t}$ sector, all evaluated in the flavor conserving MSSM.

\subsubsection{Electroweak precision observables}

EWPO that are known with an accuracy at the per-mille level or better have the potential to allow for a discrimination between quantum effects of the SM and SUSY models; see [71] for a review. Examples are the $W$-boson mass $M_{W}$ and the $Z$-boson observables, such as the effective leptonic weak mixing angle $\sin ^{2} \theta_{\text {eff }}$, whose present experimental uncertainties are [72]

$\delta M_{W}^{\text {exp,today }} \sim 15 \mathrm{MeV}, \quad \delta \sin ^{2} \theta_{\text {eff }}^{\text {exp,today }} \sim 15 \times 10^{-5}$.

The experimental uncertainty will further be reduced $[73,74]$ to

$\delta M_{W}^{\text {exp,future }} \sim 4 \mathrm{MeV}, \quad \delta \sin ^{2} \theta_{\text {eff }}^{\text {exp future }} \sim 1.3 \times 10^{-5}$

at the ILC and at the GigaZ option of the ILC, respectively. An even higher precision could be expected from the FCC-ee; see, e.g., [75].
The $W$-boson mass can be evaluated from

$M_{W}^{2}\left(1-\frac{M_{W}^{2}}{M_{Z}^{2}}\right)=\frac{\pi \alpha}{\sqrt{2} G_{\mu}}(1+\Delta r)$

where $\alpha$ is the fine-structure constant and $G_{\mu}$ the Fermi constant. This relation arises from comparing the prediction for muon decay with the experimentally precisely known Fermi constant. The one-loop contributions to $\Delta r$ can be written as

$\Delta r=\Delta \alpha-\frac{c_{\mathrm{W}}^{2}}{s_{\mathrm{w}}^{2}} \Delta \rho+(\Delta r)_{\mathrm{rem}}$,

where $\Delta \alpha$ is the shift in the fine-structure constant due to the light fermions of the SM, $\Delta \alpha \propto \log \left(M_{Z} / m_{f}\right)$, and $\Delta \rho$ is the leading contribution to the $\rho$ parameter [76] from (certain) fermion and sfermion loops (see below). The remainder part $(\Delta r)_{\text {rem }}$ contains in particular the contributions from the Higgs sector.

The effective leptonic weak mixing angle at the $Z$-boson resonance, $\sin ^{2} \theta_{\text {eff }}$, is defined through the vector and axialvector couplings $\left(g_{\mathrm{V}}^{\ell}\right.$ and $g_{\mathrm{A}}^{\ell}$ ) of leptons $(\ell)$ to the $Z$ boson, measured at the $Z$-boson pole. If this vertex is written as $i \bar{\ell} \gamma^{\mu}\left(g_{\mathrm{V}}^{\ell}-g_{\mathrm{A}}^{\ell} \gamma_{5}\right) \ell Z_{\mu}$ then

$\sin ^{2} \theta_{\text {eff }}=\frac{1}{4}\left(1-\operatorname{Re} \frac{g_{\mathrm{V}}^{\ell}}{g_{\mathrm{A}}^{\ell}}\right)$.

Loop corrections enter through higher-order contributions to $g_{\mathrm{V}}^{\ell}$ and $g_{\mathrm{A}}^{\ell}$.

Both of these (pseudo-)observables are affected by shifts in the quantity $\Delta \rho$ according to

$\Delta M_{W} \approx \frac{M_{W}}{2} \frac{c_{\mathrm{w}}^{2}}{c_{\mathrm{w}}^{2}-s_{\mathrm{w}}^{2}} \Delta \rho, \quad \Delta \sin ^{2} \theta_{\mathrm{eff}} \approx-\frac{c_{\mathrm{w}}^{2} s_{\mathrm{w}}^{2}}{c_{\mathrm{w}}^{2}-s_{\mathrm{w}}^{2}} \Delta \rho$.

The quantity $\Delta \rho$ is defined by the relation

$\Delta \rho=\frac{\Sigma_{Z}^{\mathrm{T}}(0)}{M_{Z}^{2}}-\frac{\Sigma_{W}^{\mathrm{T}}(0)}{M_{W}^{2}}$

with the unrenormalized transverse parts of the $Z$ - and $W$ boson self-energies at zero momentum, $\Sigma_{Z, W}^{\mathrm{T}}(0)$. It represents the leading universal corrections to the electroweak precision observables induced by mass splitting between partners in isospin doublets [76]. Consequently, it is sensitive to the mass-splitting effects induced by flavor mixing. The effects from flavor violation in the squark and slepton sector, entering via $\Delta \rho$ have been evaluated in $[57,69]$ and included in FeynHiggs. In particular, in [69] it has been shown that for the squark contributions $\Delta \rho$ constitutes an excellent 
approximation to $\Delta r$. We use FeynHiggs for our numerical evaluation.

Concerning the expected effects in $\Delta \rho$ some more detailed comments are in order. Within the SM the corrections to $\Delta \rho$ stem from the splitting in one $S U(2)$ doublet. Due to the mixing of various scalar fermion states the picture is slightly more involved in the MSSM. In MSSM without flavor violation the well-known results for the third generation squark contribution to $\Delta \rho$ (without flavor mixing) can be written as

$$
\begin{aligned}
\Delta \rho= & \frac{3 G_{\mu}}{8 \sqrt{2} \pi^{2}}\left[-\sin ^{2} \theta_{\tilde{t}} \cos ^{2} \theta_{\tilde{t}} F_{0}\left(m_{\tilde{t}_{1}}^{2}, m_{\tilde{t}_{2}}^{2}\right)-\sin ^{2} \theta_{\tilde{b}} \cos ^{2} \theta_{\tilde{b}}\right. \\
& \times F_{0}\left(m_{\tilde{b}_{1}}^{2}, m_{\tilde{b}_{2}}^{2}\right)+\cos ^{2} \theta_{\tilde{t}} \cos ^{2} \theta_{\tilde{b}} F_{0}\left(m_{\tilde{t}_{1}}^{2}, m_{\tilde{b}_{1}}^{2}\right) \\
& +\sin ^{2} \theta_{\tilde{b}} \cos ^{2} \theta_{\tilde{t}} F_{0}\left(m_{\tilde{t}_{1}}^{2}, m_{\tilde{b}_{2}}^{2}\right)+\sin ^{2} \theta_{\tilde{t}} \cos ^{2} \theta_{\tilde{b}} \\
& \left.\times F_{0}\left(m_{\tilde{t}_{2}}^{2}, m_{\tilde{b}_{1}}^{2}\right)+\sin ^{2} \theta_{\tilde{t}} \sin ^{2} \theta_{\tilde{b}} F_{0}\left(m_{\tilde{t}_{2}}^{2}, m_{\tilde{b}_{2}}^{2}\right)\right]
\end{aligned}
$$

with

$F_{0}\left(m_{1}^{2}, m_{2}^{2}\right)=m_{1}^{2}+m_{2}^{2}-\frac{2 m_{1}^{2} m_{2}^{2}}{m_{1}^{2}-m_{2}^{2}} \ln \left(\frac{m_{1}^{2}}{m_{2}^{2}}\right)$.

In the absence of intergenerational mixing there are only $2 \times 2$ mixing matrices to be taken into account, here parametrized by $\theta_{\tilde{t}}\left(\theta_{\tilde{b}}\right)$ in the scalar top (bottom) case. Here one can see that squarks do not need to be the $S U$ (2) partners to give a contribution to $\Delta \rho$. In particular the first two terms of Eq. (46) describe contributions from the same type (up type or down type) of scalar quarks. Going from this simple case to the one with generation mixing, one finds a contribution from all three generations, including two $6 \times 6$ mixing matrices (which are difficult to analyze analytically). For the sake of completeness, the two gauge boson self-energies are then given by (see also [69])

$$
\begin{aligned}
\Sigma_{Z Z}(0)= & \frac{e^{2}}{288 \pi^{2} s_{\mathrm{w}}^{2} c_{\mathrm{w}}^{2}}\left(-\sum_{s, t=1}^{6} \sum_{i, j=1}^{3} 2\left[\frac{1}{8} F_{0}\left(m_{\tilde{u}_{s}}^{2}, m_{\tilde{u}_{t}}^{2}\right)\right.\right. \\
& \left.+\frac{1}{4}\left(A_{0}^{\mathrm{fin}}\left(m_{\tilde{u}_{s}}^{2}\right)+A_{0}^{\mathrm{fin}}\left(m_{\tilde{u}_{t}}^{2}\right)\right)\right] \\
& \left\{3 R_{t, j}^{\tilde{u}} R_{t, j}^{\tilde{u}^{*}}-4 s_{\mathrm{w}}^{2}\left(R_{t, j}^{\tilde{u}} R_{t, j}^{\tilde{u}^{*}}+R_{t, 3+j}^{\tilde{u}} R_{t, 3+j}^{\tilde{u}^{*}}\right)\right\} \\
& \left\{3 R_{s, i}^{\tilde{u}} R_{s, i}^{\tilde{u}^{*}}-4 s_{\mathrm{w}}^{2}\left(R_{s, i}^{\tilde{u}} R_{s, i}^{\tilde{u}^{*}}+R_{s, 3+i}^{\tilde{u}} R_{s, 3+i}^{\tilde{u}^{*}}\right)\right\} \\
& -\sum_{s, t=1 i, j=1}^{6} \sum^{3} 2\left[\frac{1}{8} F_{0}\left(m_{\tilde{d}_{s}}^{2}, m_{\tilde{d}_{t}}^{2}\right)+\frac{1}{4}\left(A_{0}^{\mathrm{fin}}\left(m_{\tilde{d}_{s}}^{2}\right)\right.\right. \\
& \left.\left.+A_{0}^{\mathrm{fin}}\left(m_{\tilde{d}_{t}}^{2}\right)\right)\right] \\
& \left\{3 R_{t, j}^{\tilde{d}} R_{t, j}^{\tilde{d}^{*}}-2 s_{\mathrm{w}}^{2}\left(R_{t, j}^{\tilde{d}} R_{t, j}^{\tilde{d}^{*}}+R_{t, 3+j}^{\tilde{d}} R_{t, 3+j}^{\tilde{d}^{*}}\right)\right\}
\end{aligned}
$$

$$
\begin{aligned}
\{ & 3 R_{s, i}^{\tilde{u}} R_{s, i}^{\tilde{u}^{*}}-2 s_{\mathrm{W}}^{2}\left(R_{s, i}^{\tilde{d}} R_{s, i}^{\tilde{d}^{*}}+R_{s, 3+i}^{\tilde{d}} R_{s, 3+i}^{\tilde{d}^{*}}\right\} \\
& +\sum_{s=1}^{6} \sum_{i=1}^{3} A_{0}^{\mathrm{fin}}\left(m_{\tilde{u}_{s}}^{2}\right)\left[\left(3-4 s_{\mathrm{W}}^{2}\right)^{2} R_{s, i}^{\tilde{u}} R_{s, i}^{\tilde{u}^{*}}\right. \\
& \left.+16 s_{\mathrm{w}}^{4} R_{s, 3+i}^{\tilde{u}} R_{s, 3+i}^{\tilde{u}^{*}}\right] \\
& +\sum_{s=1}^{6} \sum_{i=1}^{3} A_{0}^{\mathrm{fin}}\left(m_{\tilde{d}_{s}}^{2}\right)\left[\left(3-2 s_{\mathrm{W}}^{2}\right)^{2} R_{s, i}^{\tilde{d}} R_{s, i}^{\tilde{d}^{*}}\right. \\
& \left.\left.+4 s_{\mathrm{W}}^{4} R_{s, 3+i}^{\tilde{d}} R_{s, 3+i}^{\tilde{d}^{*}}\right]\right) \\
\Sigma_{W W}(0)= & \frac{e^{2}}{32 \pi{ }^{2} s_{\mathrm{W}}^{2}}\left(-\sum_{s, t=1}^{6} \sum_{i, j=1}^{3} 4\left[\frac{1}{8} F_{0}\left(m_{\tilde{u}_{s}}^{2}, m_{\tilde{d}_{t}}^{2}\right)\right.\right. \\
& +\frac{1}{4}\left(A_{0}^{\mathrm{fin}}\left(m_{\tilde{u}_{s}}^{2}\right)+A_{0}^{\mathrm{fin}}\left(m_{\tilde{d}_{t}}^{2}\right)\right] R_{s, i}^{\tilde{u}} R_{t, j}^{\tilde{d}} R_{s, j}^{\tilde{u}^{*}} R_{t, i}^{\tilde{d}^{*}} \\
& +\sum_{s=1}^{6} \sum_{i=1}^{3} A_{0}^{\mathrm{fin}}\left(m_{\tilde{u}_{s}}^{2}\right) R_{s, i}^{\tilde{u}} R_{s, i}^{\tilde{u}^{*}} \\
& +\sum_{s=1}^{6} \sum_{i=1}^{3} A_{0}^{\mathrm{fin}}\left(m_{\tilde{d}_{s}}^{2}\right) R_{s, i}^{\tilde{d}} R_{s, i}^{\tilde{d}^{*}} . \\
&
\end{aligned}
$$

Here $R^{\tilde{u}}$ and $R^{\tilde{d}}$ are the $6 \times 6$ rotation matrices for the upand down-type squarks, respectively; see Eq. (28). The finite part of the one point integral function is given by

$A_{0}^{\mathrm{fin}}\left(m^{2}\right)=m^{2}\left(1-\log \frac{m^{2}}{\mu^{2}}\right)$

Here it is important to note that the corrections will come, as in Eq. (46), from states connected via $S U(2)$ as well as from "same flavor" contributions stemming from the $Z$ boson self-energy; see Eq. (45). Larger splitting between "same flavor" states due to the intergenerational mixing thus leads to the expectation of increasing contributions to $\Delta \rho$ from flavor-violation effects.

\subsubsection{B-physics observables}

We also calculate several $B$-physics observables (BPO): $\mathrm{BR}\left(B \rightarrow X_{s} \gamma\right), \mathrm{BR}\left(B_{s} \rightarrow \mu^{+} \mu^{-}\right)$and $\Delta M_{B_{s}}$. Concerning $\operatorname{BR}\left(B \rightarrow X_{s} \gamma\right)$ : included in the calculation are the most relevant loop contributions to the Wilson coefficients: (i) loops with Higgs bosons (including the resummation of large $\tan \beta$ effects [77]), (ii) loops with charginos, and (iii) loops with gluinos. For $\operatorname{BR}\left(B_{s} \rightarrow \mu^{+} \mu^{-}\right)$there are three types of relevant one-loop corrections contributing to the relevant Wilson coefficients: (i) box diagrams, (ii) $Z$-penguin diagrams, and (iii) neutral Higgs-boson $\phi$ penguin diagrams, where $\phi$ denotes the three neutral MSSM Higgs bosons, $\phi=h, H, A$ (again large resummed $\tan \beta$ effects have been taken into account). In our numerical eval- 
Table 1 Present experimental status of $B$-physics observables with their SM prediction

\begin{tabular}{lll}
\hline Observable & Experimental value & SM prediction \\
\hline $\mathrm{BR}\left(B \rightarrow X_{s} \gamma\right)$ & $3.43 \pm 0.22 \times 10^{-4}$ & $3.15 \pm 0.23 \times 10^{-4}$ \\
$\mathrm{BR}\left(B_{s} \rightarrow \mu^{+} \mu^{-}\right)$ & $(3.0)_{-0.9}^{+1.0} \times 10^{-9}$ & $3.23 \pm 0.27 \times 10^{-9}$ \\
$\Delta M_{B_{s}}$ & $116.4 \pm 0.5 \times 10^{-10} \mathrm{MeV}$ & $(117.1)_{-16.4}^{+17.2} \times 10^{-10} \mathrm{MeV}$ \\
\hline
\end{tabular}
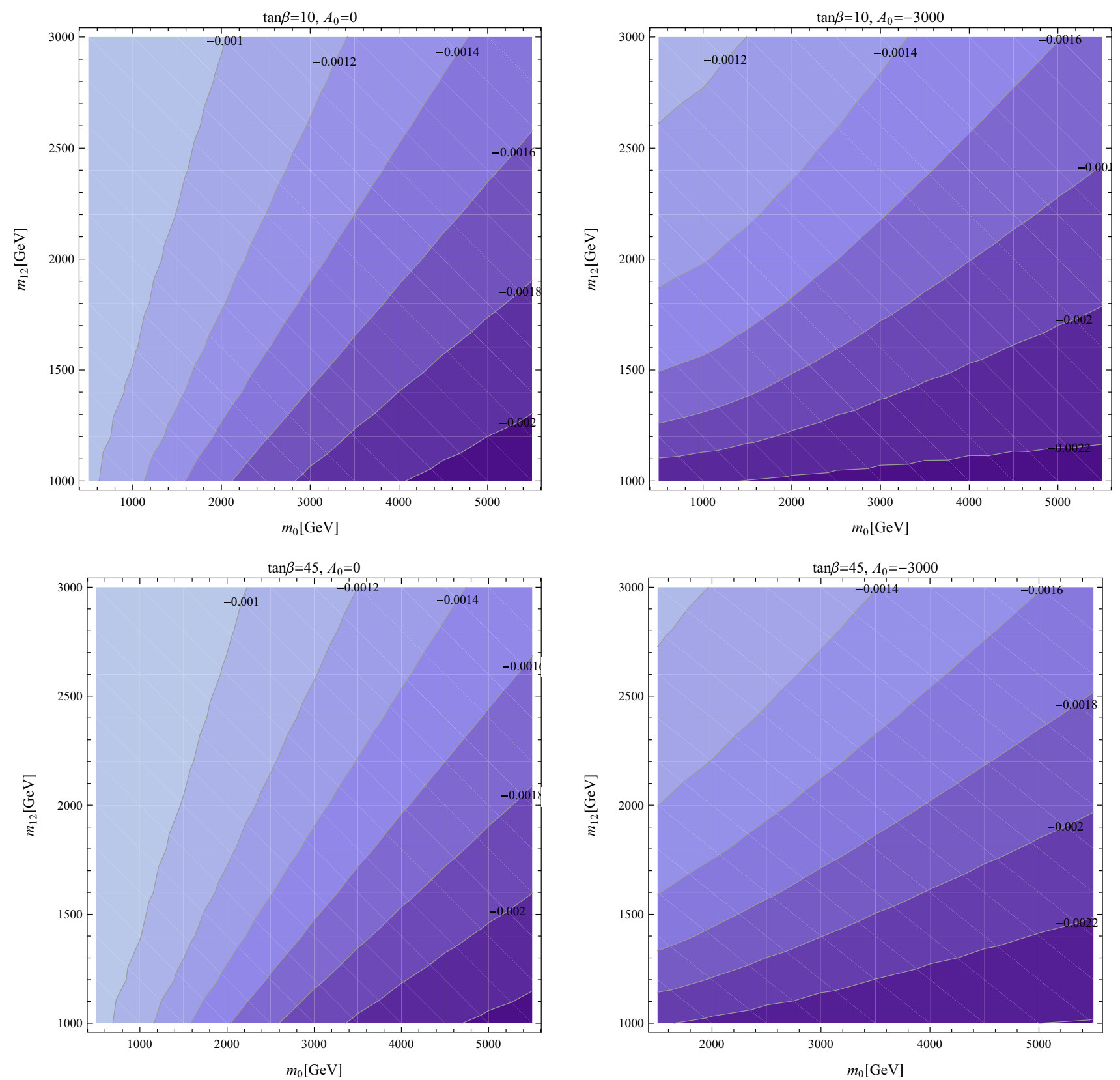

Fig. 1 Contours of $\delta_{13}^{Q L L}$ in the $m_{0}-m_{1 / 2}$ plane for different values of $\tan \beta$ and $A_{0}$ in the CMSSM

uation there are included what are known to be the dominant contributions to these three types of diagrams [78]: chargino contributions to box and $Z$-penguin diagrams, and chargino and gluino contributions to $\phi$-penguin diagrams. Concerning $\Delta M_{B_{s}}$, in the MSSM there are in general three types of one-loop diagrams that contribute: (i) box diagrams, 

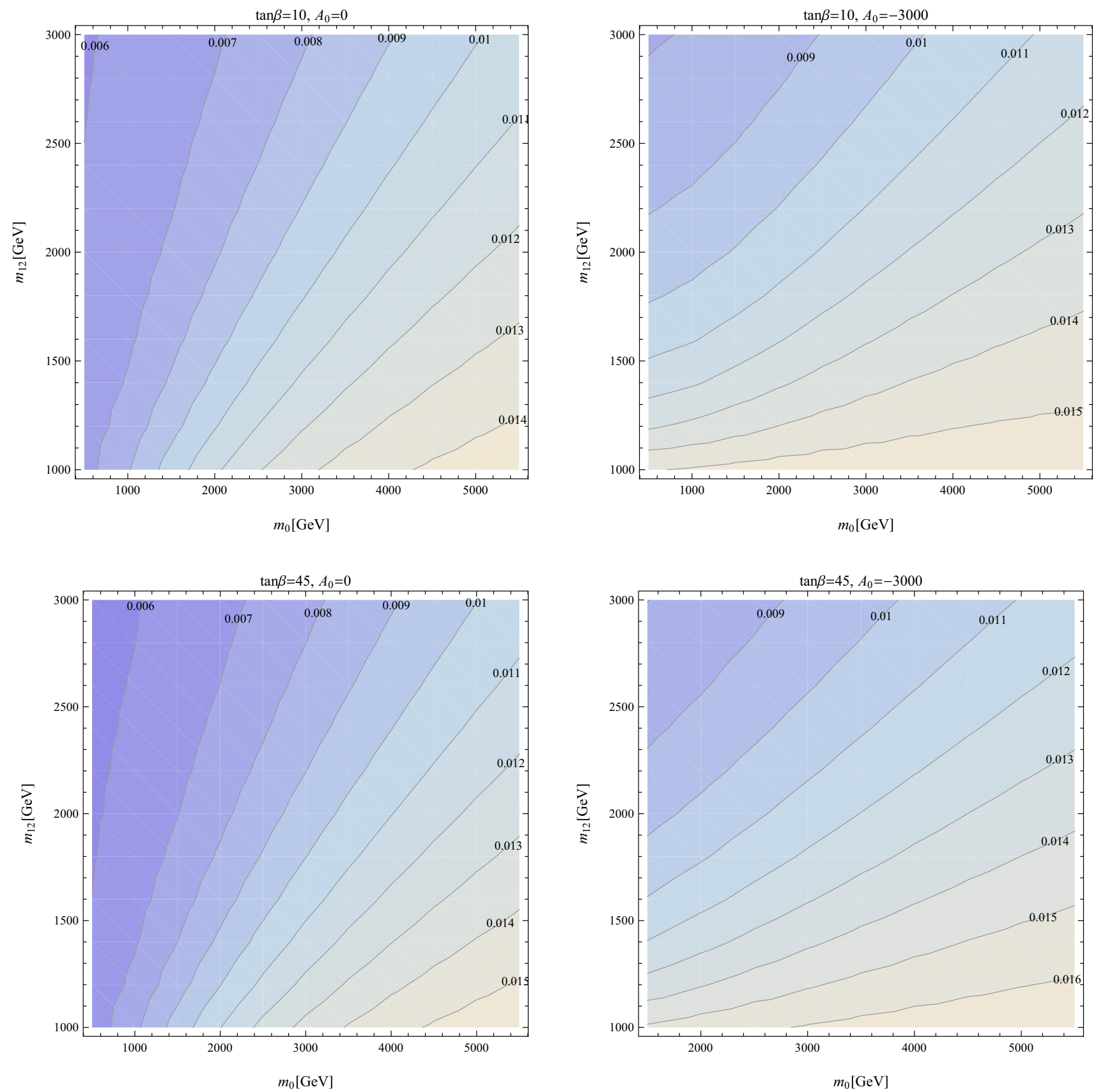

Fig. 2 Contours of $\delta_{23}^{Q L L}$ in the $m_{0}-m_{1 / 2}$ plane for different values of $\tan \beta$ and $A_{0}$ in the CMSSM

(ii) Z-penguin diagrams, and (iii) double Higgs-penguin diagrams (again including the resummation of large $\tan \beta$ enhanced effects). In our numerical evaluation there are included again what are known to be the dominant contributions to these three types of diagrams in scenarios with non-minimal flavor violation (for a review see, for instance, [79]): gluino contributions to box diagrams, chargino contributions to box and $Z$-penguin diagrams, and chargino and gluino contributions to double $\phi$-penguin diagrams. More details about the calculations employed can be found in [44-46]. We perform our numerical calculation with the BPHYSICS subroutine taken from the SuFla code $[42,43]$ (with some additions and improvements as detailed in [4446]), which has been implemented as a subroutine into (a private version of) FeynHiggs. The present experimental status and SM prediction of these observables is given in Table 1 [80-87]. 

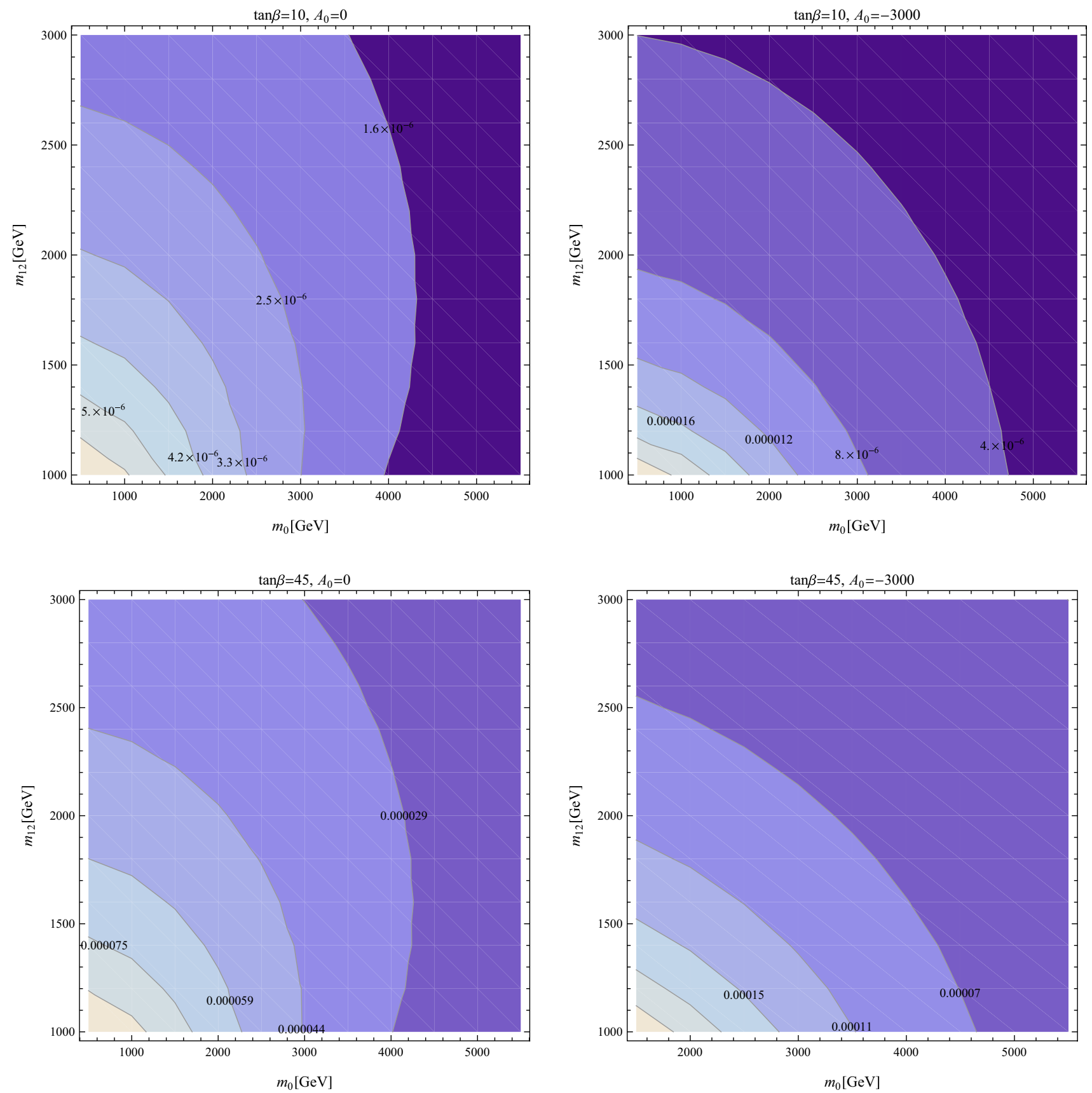

Fig. 3 Contours of $\delta_{23}^{U L R}$ in the $m_{0}-m_{1 / 2}$ plane for different values of $\tan \beta$ and $A_{0}$ in the CMSSM

\section{Numerical results}

\subsection{Effects of squark mixing in the CMSSM}

In this section we analyze the effects from RGE induced flavor-violating mixing in the scalar quark sector in the CMSSM (i.e. with no mixing in the slepton sector). The RGE running from the GUT scale to the EW has been performed as described in Sect. 3.1, with the subsequent evaluation of the low-energy observables as discussed in Sect. 3.2. In order to get an overview of the size of the effects in the CMSSM parameter space, the relevant parameters $m_{0}, m_{1 / 2}$ have been scanned as (or in the case of $A_{0}$ and $\tan \beta$ have been set to) all combinations of

$$
\begin{aligned}
m_{0} & =500 \mathrm{GeV} \ldots 5000 \mathrm{GeV}, \\
m_{1 / 2} & =1000 \mathrm{GeV} \ldots 3000 \mathrm{GeV}, \\
A_{0} & =-3000,-2000,-1000,0 \mathrm{GeV},
\end{aligned}
$$



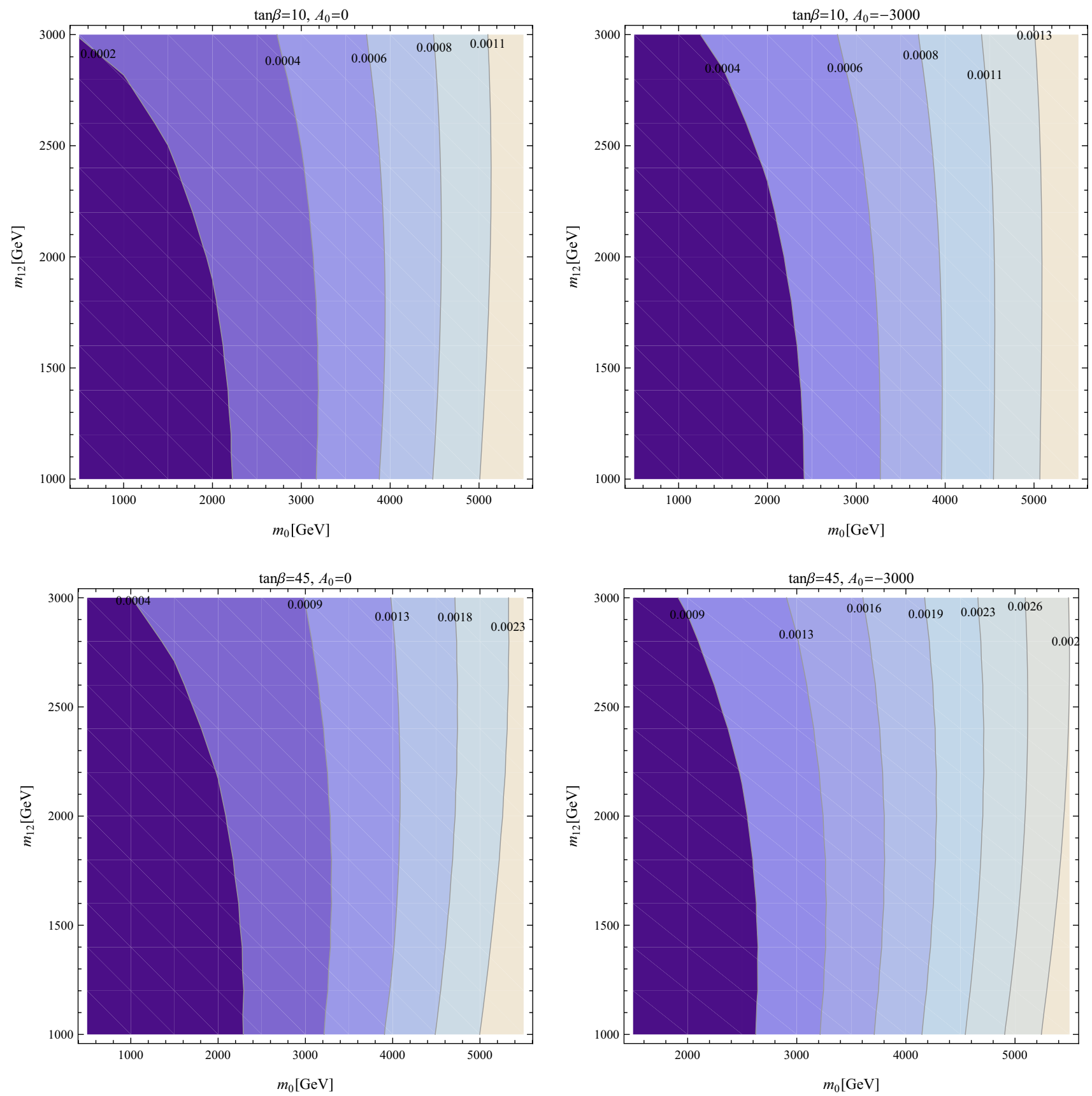

Fig. 4 Contours of $\Delta \rho^{\mathrm{MFV}}$ in the $m_{0}-m_{1 / 2}$ plane for different values of $\tan \beta$ and $A_{0}$ in the CMSSM

$\tan \beta=10,20,35,45$,

with $\mu>0$. Primarily we are not interested in the absolute values for all these observables but the effects that come from flavor violation within the MFV framework, i.e. the effect from the off-diagonal entries in the sfermion mass matrices. We first calculate the low-energy observables by setting all $\delta_{i j}^{F A B}=0$ by hand. In a second step we evaluate the observables with the values of $\delta_{i j}^{F A B}$ obtained through RGE running.
We then evaluate the "pure MFV effects",

$$
\begin{aligned}
& \Delta \mathrm{BR}^{\mathrm{MFV}}\left(B \rightarrow X_{s} \gamma\right) \\
& \left.\quad=\mathrm{BR}\left(B \rightarrow X_{s} \gamma\right)-\mathrm{BR}^{\mathrm{MSSM}}\right)\left(B \rightarrow X_{s} \gamma\right), \\
& \Delta \mathrm{BR}^{\mathrm{MFV}}\left(B_{s} \rightarrow \mu^{+} \mu^{-}\right) \\
& \quad=\mathrm{BR}\left(B_{s} \rightarrow \mu^{+} \mu^{-}\right)-\mathrm{BR}^{\mathrm{MSSM}}\left(B_{s} \rightarrow \mu^{+} \mu^{-}\right), \\
& \Delta M_{B_{s}}^{\mathrm{MFV}}=\Delta M_{B_{s}}-\Delta M_{B_{s}}^{\mathrm{MSSM}},
\end{aligned}
$$



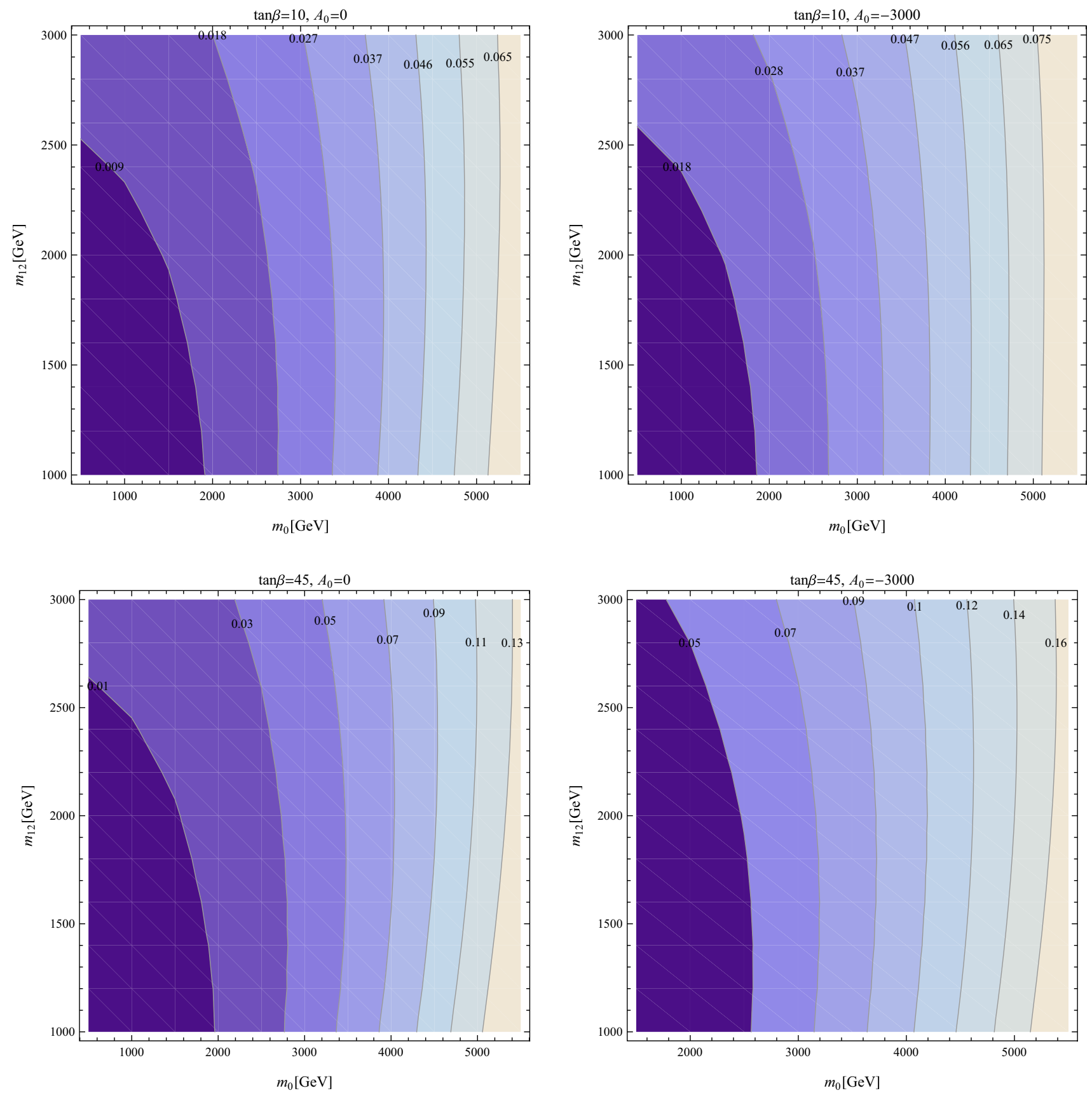

Fig. 5 Contours of $\Delta M_{W}^{\mathrm{MFV}}$ in GeV in the $m_{0}-m_{1 / 2}$ plane for different values of $\tan \beta$ and $A_{0}$ in the CMSSM

where $\mathrm{BR}^{\mathrm{MSSM}}\left(B \rightarrow X_{s} \gamma\right), \mathrm{BR}^{\mathrm{MSSM}}\left(B_{s} \rightarrow \mu^{+} \mu^{-}\right)$, and $\Delta M_{B S}^{\mathrm{MSSM}}$ corresponds to the values of relevant observables with all $\delta_{i j}^{F A B}=0$. Furthermore we use

$$
\begin{aligned}
\Delta M_{h}^{\mathrm{MFV}} & =M_{h}-M_{h}^{\mathrm{MSSM}}, \\
\Delta M_{H}^{\mathrm{MFV}} & =M_{H}-M_{H}^{\mathrm{MSSM}}, \\
\Delta M_{H^{ \pm}}^{\mathrm{MFV}} & =M_{H^{ \pm}}-M_{H^{ \pm}}^{\mathrm{MSSM}},
\end{aligned}
$$

where $M_{h}^{\mathrm{MSSM}}, M_{H}^{\mathrm{MSSM}}$, and $M_{H^{ \pm}}^{\mathrm{MSSM}}$ corresponds to the Higgs masses with all $\delta_{i j}^{F A B}=0$. Similarly we use for the EWPO

$$
\begin{aligned}
\Delta \rho^{\mathrm{MFV}} & =\Delta \rho-\Delta \rho^{\mathrm{MSSM}}, \\
\Delta M_{W}^{\mathrm{MFV}} & =M_{W}-M_{W}^{\mathrm{MSSM}}, \\
\Delta \sin ^{2} \theta_{\mathrm{eff}}^{\mathrm{MFV}} & =\sin ^{2} \theta_{\mathrm{eff}}-\sin ^{2} \theta_{\mathrm{eff}}^{\mathrm{MSSM}},
\end{aligned}
$$



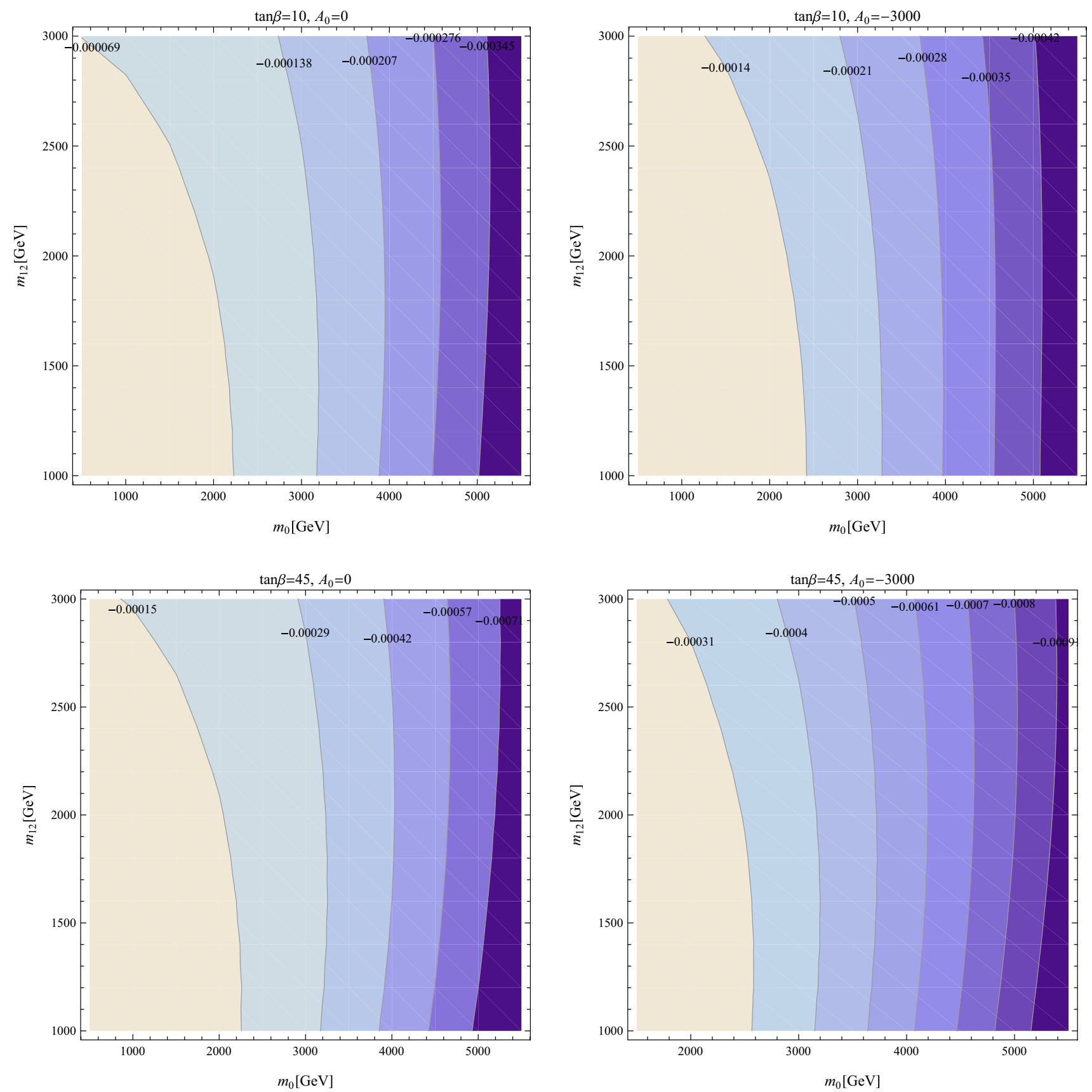

Fig. 6 Contours of $\Delta \sin ^{2} \theta_{\text {eff }}^{\mathrm{MFV}}$ in the $m_{0}-m_{1 / 2}$ plane for different values of $\tan \beta$ and $A_{0}$ in the CMSSM

where $\Delta \rho^{\mathrm{MSSM}}, M_{W}^{\mathrm{MSSM}}$, and $\sin ^{2} \theta_{\text {eff }}^{\mathrm{MSSM}}$ are the values of the relavant observables with all $\delta_{i j}^{F A B}=0$.

In Figs. 1, 2, 3, 4, 5, 6, 7 and 8 we show the results of our CMSSM analysis in the $m_{0}-m_{1 / 2}$ plane for four different combinations of $\tan \beta=10,45$ (left and right column) and $A_{0}=0,-3000 \mathrm{GeV}$ (upper and lower row). This set represents four "extreme" cases of the parameter space and give an overview about the possible sizes of the effects and their dependences on $\tan \beta$ and $A_{0}$ (which we verified with other, not shown, combinations). We start with the three most relevant $\delta_{i j}^{F A B}$. In Figs. 1, 2 and 3 we show the results for $\delta_{13}^{Q L L}, \delta_{23}^{Q L L}$, and $\delta_{23}^{U L R}$, respectively, which are expected to yield the largest results. The values show the expected pattern of their size with $\delta_{23}^{Q L L} \sim \mathcal{O}\left(10^{-2}\right)$ being the largest one, and $\delta_{13}^{Q L L}$ and $\delta_{23}^{U L R}$ about one or two orders of magnitude smaller. All other $\delta_{i j}^{F A B}$, which are not shown, reach only values of $\mathcal{O}\left(10^{-5}\right)$. One can observe an interesting pattern in these figures: the values of $\delta_{i j}^{F A B}$ increase with larger values of either $\tan \beta$ or $A_{0}$. The values for $\delta^{Q L L}$ increase with $m_{0}$, 


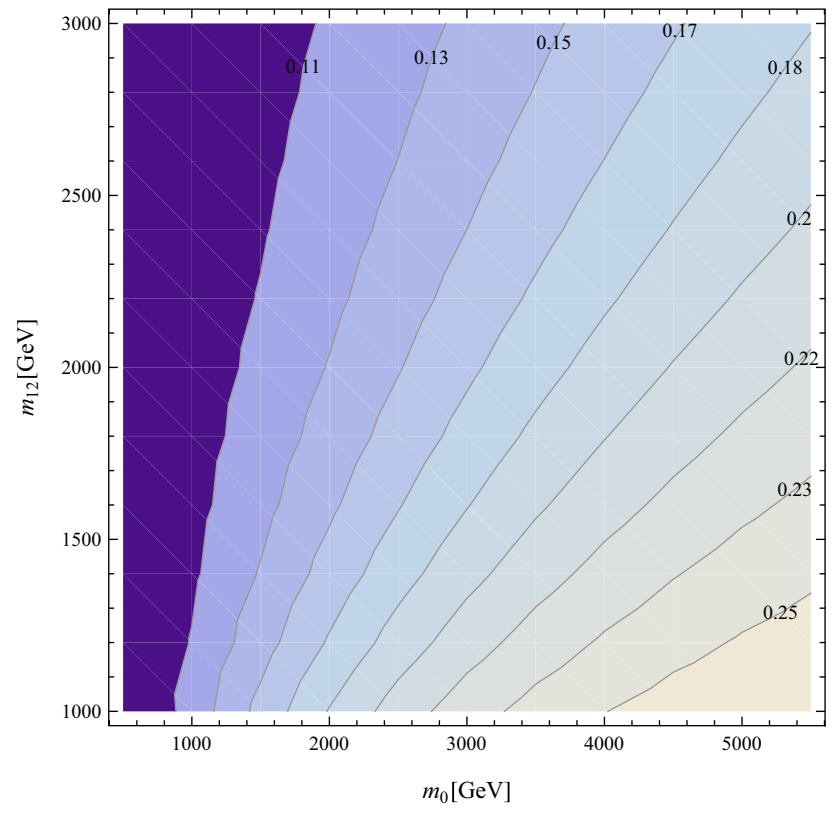

Fig. 7 Contours of $\left(m_{2}^{2}-m_{1}^{2}\right) /\left(m_{2}^{2}+m_{1}^{2}\right)$ in the $m_{0}-m_{1 / 2}$ plane for fixed values of $A_{0}=0$ and $\tan \beta=45$. Left the two most stop-like squarks (i.e. in the limit of zero intergenerational mixing they coincide

whereas $\delta^{U L R}$ and $\delta^{D L R}$ decrease with $m_{0}$. This behavior can be understood for the RGEs of the non-diagonal SUSY breaking parameters (see, e.g., [88,89]), the $\delta^{Q L L}$ are defined as ratios of off-diagonal soft terms that grow with $m_{0}^{2}$ over diagonal soft masses that also grow with $m_{0}$. However, the $\delta^{U L R}$ and the $\delta^{D L R}$ arise from the ratio of the RGE generated off-diagonal trilinear terms, which depend on the value of $A_{0}$, which is considered fixed in our case, over diagonal soft masses growing with $m_{0}$. As discussed above, these $\delta_{i j}^{F A B} \neq 0$ are often neglected in phenomenological analyses of the CMSSM (see, e.g., [29-32]). We also emphasize that these effects are purely due to the presence of the CKM matrix on the RGEs; their contribution will vanish when the mixing of the two first generation with the third generation is neglected (as we have checked numerically).

In Figs. 4, 5 and 6 we analyze the effects of the nonzero $\delta_{i j}^{F A B}$ on the EWPO $\Delta \rho^{\mathrm{MFV}}, \Delta M_{W}^{\mathrm{MFV}}$ and $\Delta \sin ^{2} \theta_{\mathrm{eff}}^{\mathrm{MFV}}$, respectively. Here the same pattern is reflected for the EWPO, i.e. by increasing the value of $\tan \beta$ or $A_{0}$, we find larger contributions to the EWPO. In particular, one can observe a nondecoupling effect for large values of $m_{0}$. Larger soft SUSYbreaking parameters with the non-zero values in particular of $\delta_{23}^{Q L L}$, see above, lead to an enhanced splitting in masses belonging to an $S U$ (2) doublet, and thus to an enhanced contribution to the $\rho$-parameter. The corresponding effects on $M_{W}$ and $\sin ^{2} \theta_{\text {eff }}$, for $m_{0} \gtrsim 3 \mathrm{TeV}$, exhibit corrections that are several times larger than the current experimental accuracy (whereas the SUSY corrections with all $\delta_{i j}^{F A B}=0$ decouple and go to zero). Consequently, including the non-

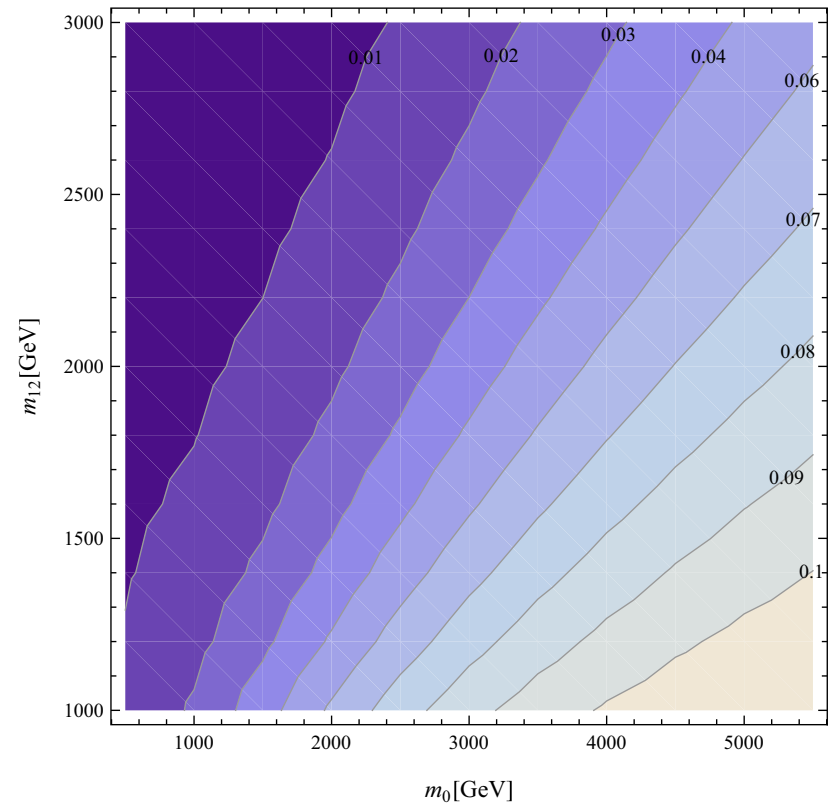

with the two scalar tops), right the lightest most stop-like and most sbottom-like squarks (see text)

zero values of the $\delta_{i j}^{F A B}$ and correctly taking these corrections into account, would yield an upper limit on $m_{0}$, which in the known analyses so far is unconstrained from above [29-32]. A more detailed analysis within the CMSSM will be needed to determine the real upper bound on $m_{0}$, which, however, is beyond the scope of this paper.

In order to gain more insight about the source of the large corrections to $\Delta \rho$ (and thus to the EWPO), we show in Fig. 7 several relative mass (square) differences, $\left(m_{2}^{2}-m_{1}^{2}\right) /\left(m_{2}^{2}+\right.$ $m_{1}^{2}$ ) in the $m_{0}-m_{1 / 2}$ plane for fixed $A_{0}=0$ and $\tan \beta=45$. The left plot shows the mass difference for the two most stop-like squarks (i.e. in the limit of zero intergenerational mixing they coincide with the two scalar tops). The right plot shows the relative mass difference for the lightest most stoplike and most sbottom-like squark. (These results are simply the Spheno output in our scenario.) In both cases one can see that the relative mass differences increase (controlled by the non-zero $\delta_{i j}^{F A B}$ induced by the CKM matrix in the RGE running) in a fashion similar to the $\delta Q L L$ discussed above, i.e. in particular for $m_{0}>m_{1 / 2}>1 \mathrm{TeV}$. These increasing mass differences lead (together with contributions from the mixing matrices) to the observed increase of $\Delta \rho$ as in Fig. 4 .

Our findings can be briefly compared to the existing literature. The EWPO in the context of flavor violation were evaluated first in [69], where correspondingly large corrections were found for large $\delta_{23}^{Q L L}$ (in fact, that was the only parameter dependence analyzed in that paper, and only the mixing between the second and third generation of squarks was taken into account). Subsequently, the EWPO were also 

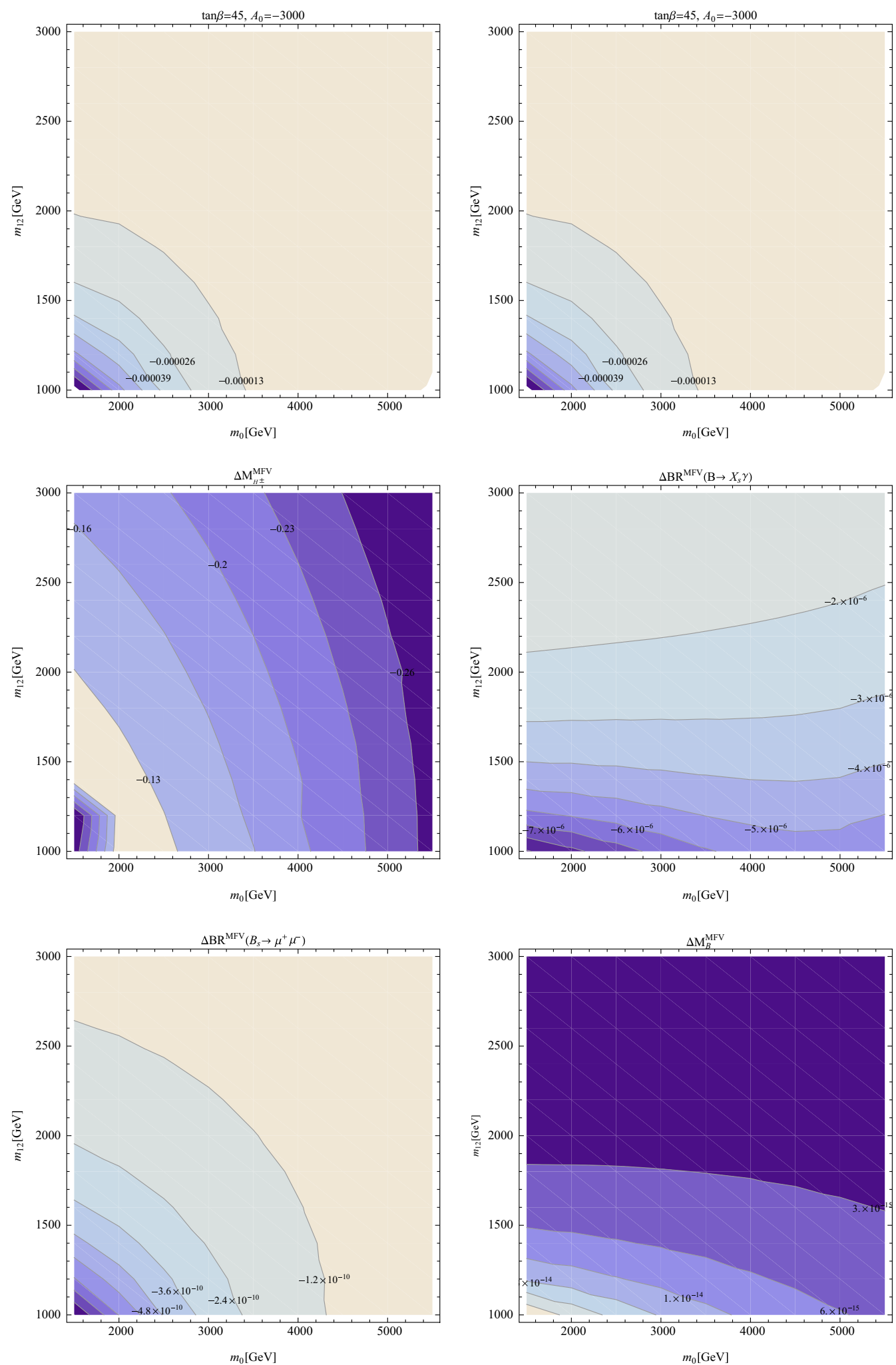

Fig. 8 Contours of Higgs mass corrections $\left(\Delta M_{h}^{\mathrm{MFV}}, \Delta M_{H}^{\mathrm{MFV}}\right.$ and $\Delta M_{H^{ \pm}}^{\mathrm{MFV}}$ in GeV) and $\mathrm{BPO}\left(\Delta \mathrm{BR}^{\mathrm{MFV}}\left(B \rightarrow X_{s} \gamma\right), \Delta \mathrm{BR}^{\mathrm{MFV}}\left(B_{s} \rightarrow \mu^{+} \mu^{-}\right)\right.$ and $\Delta M_{B_{S}}^{\mathrm{MFV}}$ ) in the $m_{0}-m_{1 / 2}$ plane for $\tan \beta=45$ and $A_{0}=-3000 \mathrm{GeV}$ in the CMSSM 

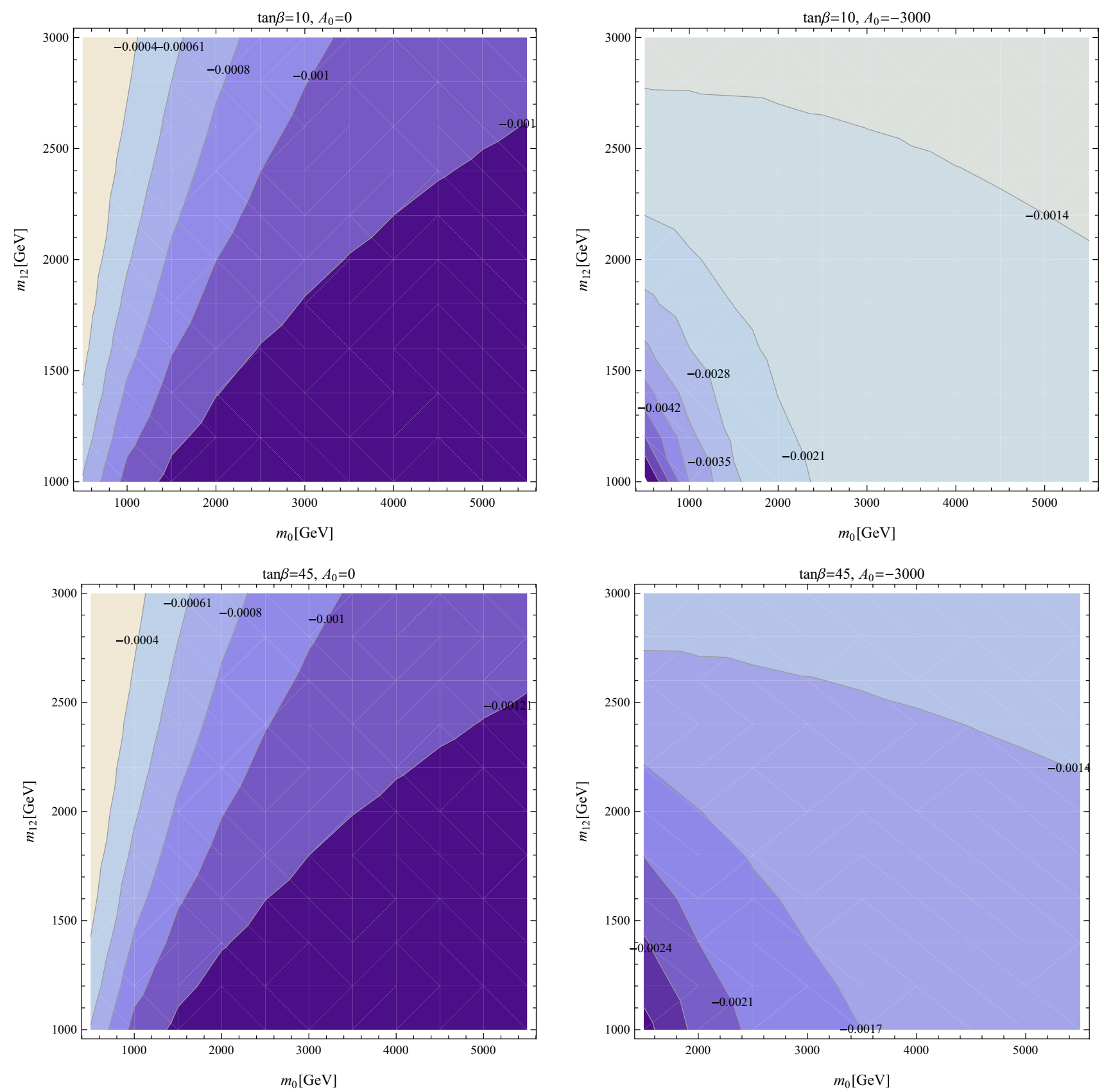

Fig. 9 Contours of $\delta_{12}^{L L L}$ in the $m_{0}-m_{1 / 2}$ plane for different values of $\tan \beta$ and $A_{0}$ in the CMSSM-seesaw I

evaluated for the full three-generation mixing in [90]. The numerical analysis, however, was restricted to a degenerate and fixed SUSY mass scale. Correspondingly, no large effects with increasing SUSY mass scales were analyzed and only relative small corrections were found. Due to the different numerical setup, however, there is no contradiction with our results for $\Delta \rho$.

In Fig. 8 we show the results of our CMSSM analysis with the effects of the non-zero $\delta_{i j}^{F A B}$ on the Higgs mass calculations and on the BPO in the $m_{0}-m_{1 / 2}$ plane for $\tan \beta=45$ and $A_{0}=-3000$. We only show this "extreme" case, where smaller values of $\tan \beta$ and $A_{0}$ would lead to smaller effects. In the upper left, upper right and middle left plot we show $\Delta M_{h}^{\mathrm{MFV}}, \Delta M_{H}^{\mathrm{MFV}}$ and $\Delta M_{H^{ \pm}}^{\mathrm{MFV}}$, respectively. It can be seen that the effects on the neutral Higgs-boson masses are negligible w.r.t. the experimental accuracy. The effects on $M_{H^{ \pm}}$ can reach $\mathcal{O}(100 \mathrm{MeV})$, where largest effects are found for both very small values of $m_{0}$ and $m_{1 / 2}$ (dominated by $\delta_{23}^{U L R}$ ) or very large values of $m_{0}$ and $m_{1 / 2}$ (dominated by $\delta_{13,23}^{Q L L}$ ). Corrections of up to $-300 \mathrm{MeV}$ are found, but still remain- 

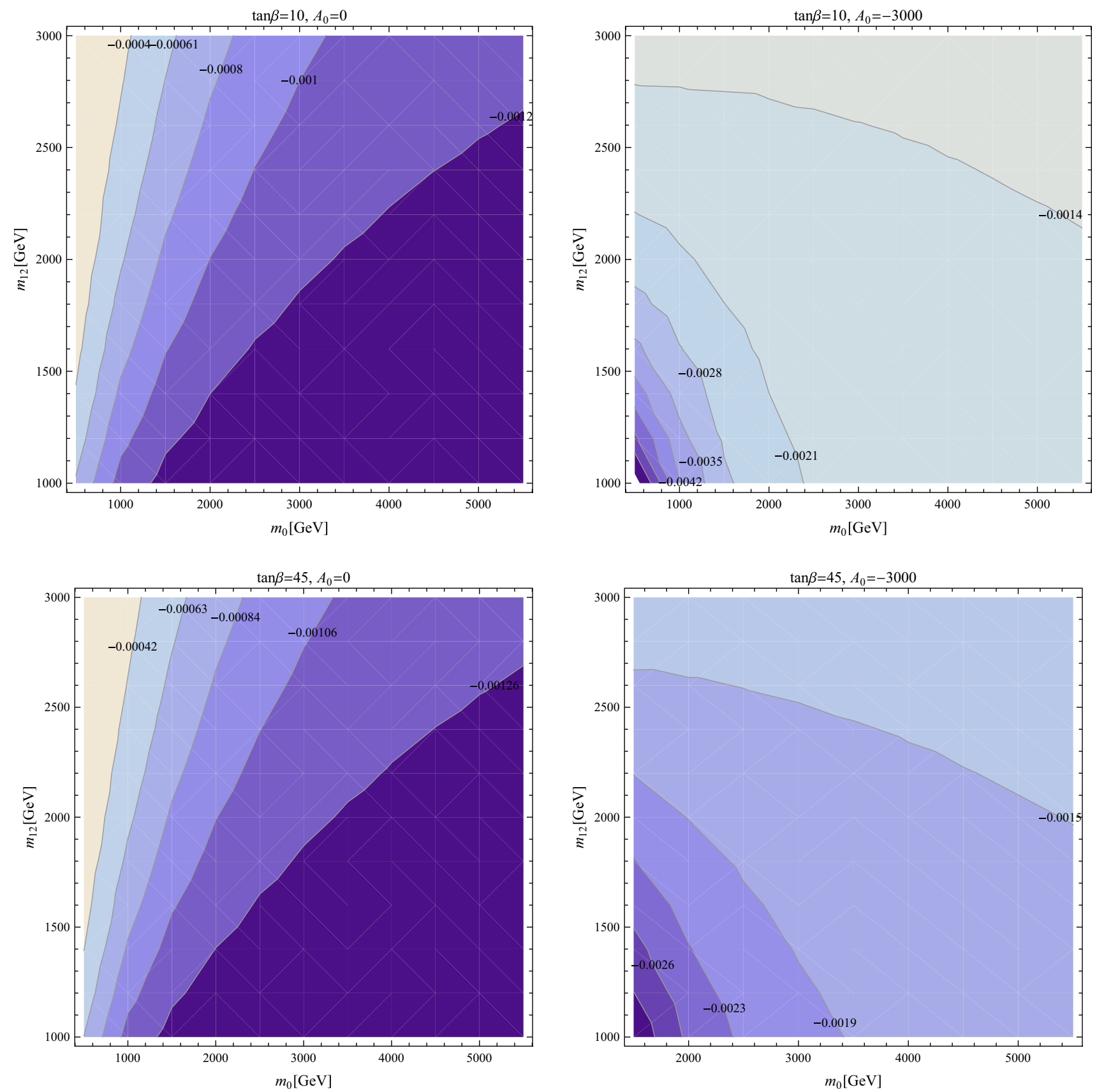

Fig. 10 Contours of $\delta_{13}^{L L L}$ in the $m_{0}-m_{1 / 2}$ plane for different values of $\tan \beta$ and $A_{0}$ in the CMSSM-seesaw I

ing below the foreseeable future precision. Consequently, also in the Higgs mass evaluation not taking into account the non-zero values of the $\delta_{i j}^{F A B}$ is a good approximation.

In the middle right, lower left, and lower right plot of Fig. 8 we show the results for the $\mathrm{BPO} \Delta \mathrm{BR}^{\mathrm{MFV}}\left(B \rightarrow X_{s} \gamma\right)$, $\Delta \mathrm{BR}^{\mathrm{MFV}}\left(B_{s} \rightarrow \mu^{+} \mu^{-}\right)$and $\Delta M_{B_{s}}^{\mathrm{MFV}}$, respectively. The effects in $\Delta \mathrm{BR}^{\mathrm{MFV}}\left(B \rightarrow X_{s} \gamma\right)$ are of $\mathcal{O}\left(-10^{-5}\right)$ and thus one order of magnitude smaller than the experimental accuracy. Similarly, we find $\Delta \mathrm{BR}^{\mathrm{MFV}}\left(B_{s} \rightarrow \mu^{+} \mu^{-}\right) \sim$ $\mathcal{O}\left(10^{-10}\right)$ and $\Delta M_{B_{s}}^{\mathrm{MFV}} \sim \mathcal{O}\left(10^{-15} \mathrm{GeV}\right)$, i.e. one or several orders of magnitude below the experimental precision. This shows that for the BPO neglecting the effects of non-zero $\delta_{i j}^{F A B}$ in the CMSSM is a good approximation.

\subsection{Effects of slepton mixing}

In this section we analyze the effects of non-zero $\delta_{i j}^{F A B}$ values in the CMSSM-seesaw I. In order to investigate the effects induced just by the mixings in the slepton sector, such that we can compare their contribution from the one produced 

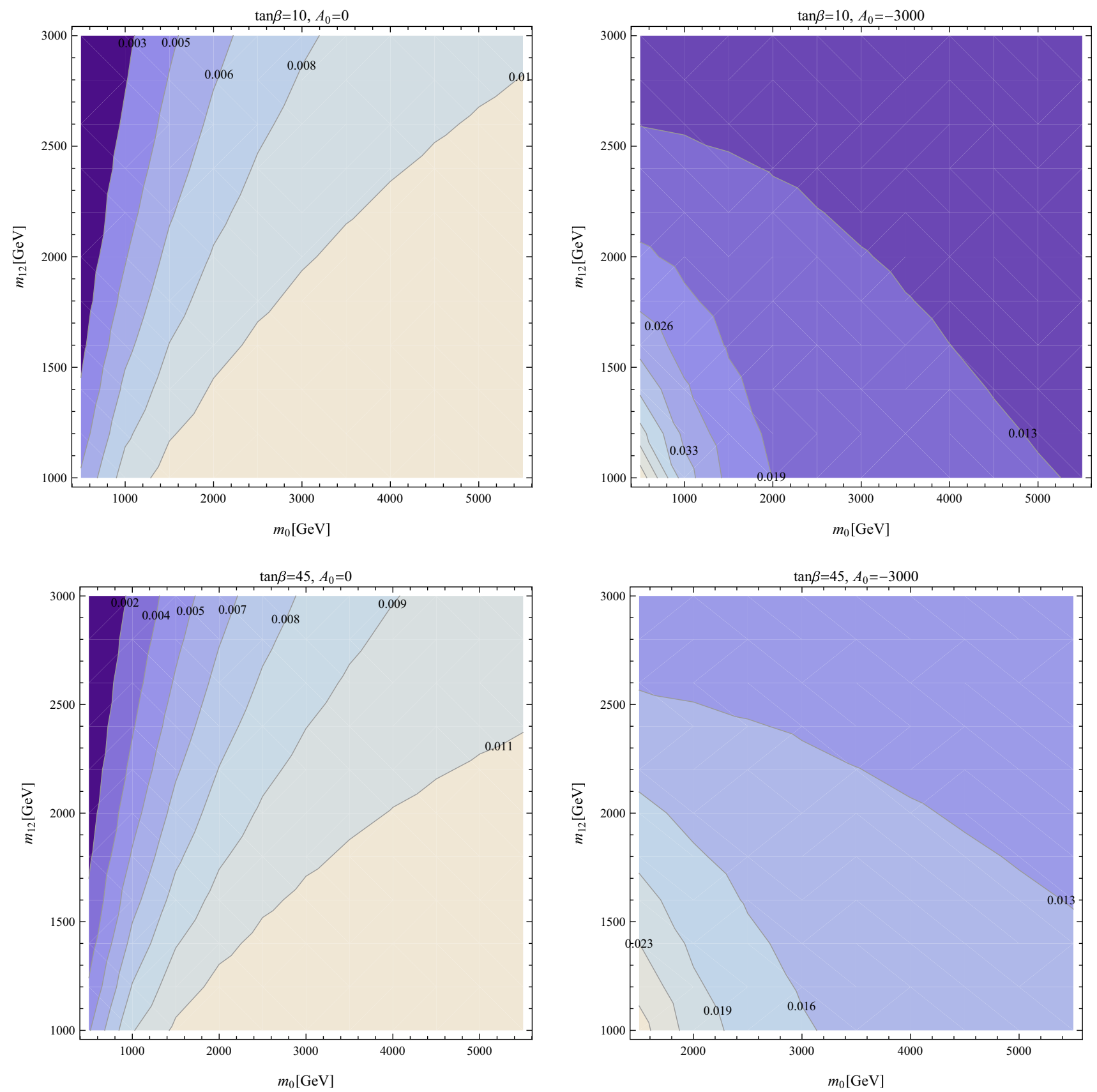

Fig. 11 Contours of $\delta_{23}^{L L L}$ in the $m_{0}-m_{1 / 2}$ plane for different values of $\tan \beta$ and $A_{0}$ in the CMSSM-seesaw I

by the mixings in the squak sector (and to discriminate it from effects from mixings in the squark sector) we present here the results with only $\delta_{i j}^{F A B}$ in the slepton sector nonzero, i.e. after the RGE running with both CKM and seesaw parameters non-zero, the $\delta_{i j}^{F A B}$ from the squark sector are set to zero by hand at the EW scale. The effects of the squark mixing in the CMSSM-seesaw I are nearly indistinguishable from the ones analyzed in the previous subsection.

As mentioned in Sect. 2.2, the calculations in this section are done by using the values of $Y_{v}$ constructed from Eq. (10) with the degenerate $M_{R}$. The matrix $R$ is set to the identity since it does not enter in Eq. (12) and therefore the slepton $\delta_{i j}^{F A B}$ do not depend on it. The matrix $m_{\nu}^{\delta}$ is a diagonal mass matrix adjusted to reproduce neutrino masses at low energy compatible with the experimental observations and with hierarchical neutrino masses. We performed our computation by using the seesaw scale $M_{N}=10^{14} \mathrm{GeV}$. With this choice the bound $B R(\mu \rightarrow e \gamma)<5.7 \times 10^{-13}$ [91] imposes severe restrictions on the $m_{0}-m_{1 / 2}$ plane, excluding values of $m_{0}$ below $2-3 \mathrm{TeV}$ (depending on $\tan \beta$ and $A_{0}$ ). The values of 

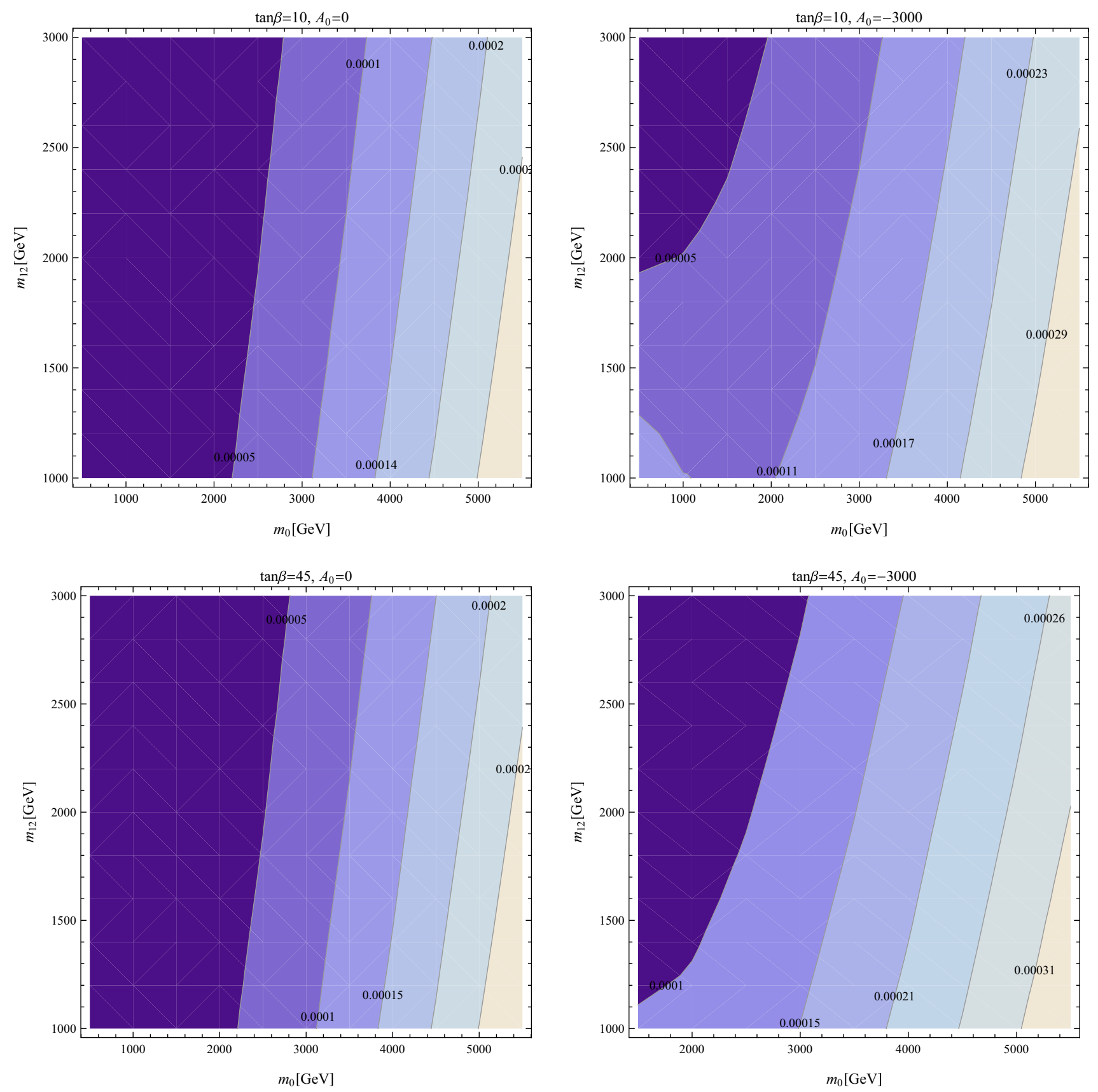

Fig. 12 Contours of $\Delta \rho^{\mathrm{MFV}}$ in the $m_{0}-m_{1 / 2}$ plane for different values of $\tan \beta$ and $A_{0}$ in the CMSSM-seesaw I

the slepton $\delta_{i j}^{F A B}$ will increase as the scale $M_{N}$ increases but the parameter space excluded by the $\operatorname{BR}(\mu \rightarrow e \gamma)$ bound will also increase. For example, by increasing $M_{N}$ by an order of magnitude, the largest entries in the matrix $Y_{v}$ will become of $\mathcal{O}(1)$ and the bound on $\operatorname{BR}(\mu \rightarrow e \gamma)$ will only be satisfied if $m_{0} \approx 5 \mathrm{TeV}$.

Our numerical results in the CMSSM-seesaw I are shown in Figs. 9, 10, 11, 12, 13, 14 and 15. As in the CMSSM we present the results in the $m_{0}-m_{1 / 2}$ plane for four combinations of $\tan \beta=10,45$ (upper and lower row) and
$A_{0}=0,-3000 \mathrm{GeV}$ (left and right column), again capturing the "extreme" cases. We start presenting the three most relevant $\delta_{i j}^{F A B}$. Figures 9,10 and 11 show $\delta_{12}^{L L L}, \delta_{13}^{L L L}$, and $\delta_{23}^{L L L}$, respectively. As expected, $\delta_{23}^{L L L}$ turns out to be largest of $\mathcal{O}(0.01)$, while the other two are about one order of magnitude smaller. The dependence on $\tan \beta$ is not very prominent, but going from $A_{0}=0$ to $-3000 \mathrm{GeV}$ has a strong impact on the $\delta_{i j}^{F A B}$. For small $A_{0}$ the size of the $\delta_{i j}^{F A B}$ is increasing with larger $m_{0}$ and $m_{1 / 2}$, for $A_{0}=-3000 \mathrm{GeV}$ the largest values are found for small $m_{0}$ and $m_{1 / 2}$. Here 

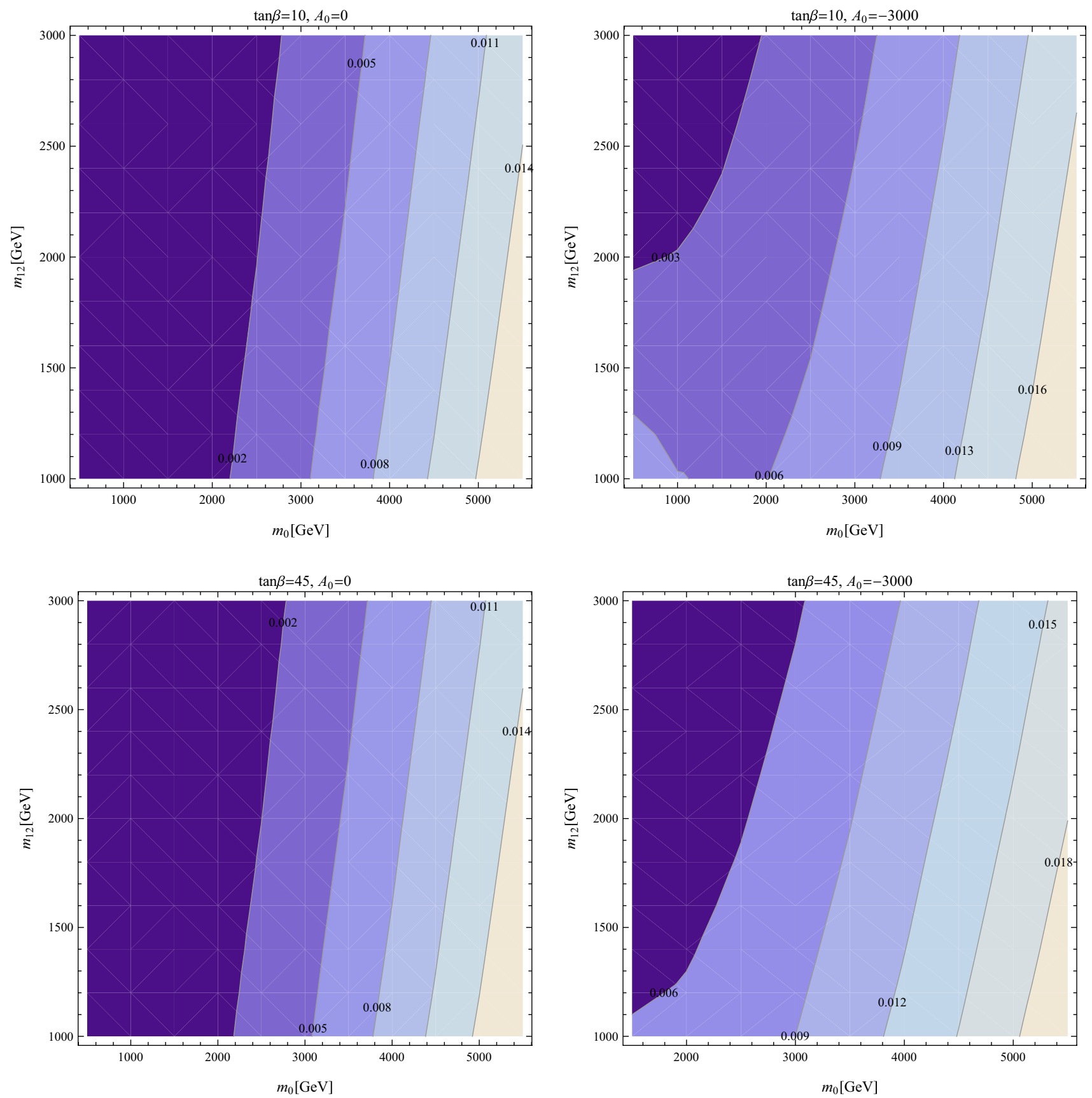

Fig. 13 Contours of $\Delta M_{W}^{\mathrm{MFV}}$ in GeV in the $m_{0}-m_{1 / 2}$ plane for different values of $\tan \beta$ and $A_{0}$ in the CMSSM-seesaw I

one comment on flavor-violating decays is in order. The selected values of $Y_{v}$ result in a large prediction for, e.g., $\mathrm{BR}(\mu \rightarrow e \gamma)$ that can eliminate some of the $m_{0}-m_{1 / 2}$ parameter plane, in particular combinations of low values of $m_{0}$ and $m_{1 / 2}$. For our parameter settings these regions are small for $\tan \beta=10$ and reach up to roughly $m_{0}+m_{1 / 2} \sim 2000 \mathrm{GeV}$ for $A_{0}=-3000 \mathrm{GeV}$. For $\tan \beta=45$ they are larger and exclude roughly the lower left half of the $m_{0}-m_{1 / 2}$ planes shown.
In Figs. 12, 13 and 14 we show the results for the EWPO. The same pattern and non-decoupling behavior for EWPO as in the case of CMSSM (squark $\delta_{i j}^{F A B}$ ) can be observed. However, the corrections induced by slepton-flavor violation are relatively small compared to squark case. For the most extreme cases, i.e. the largest values of $m_{0}$, the corrections to $M_{W}$ turn out to be of the same order of the experimental uncertainty. For those parts of the parameter space neglecting the effects of LFV to the EWPO could turn out to be an 

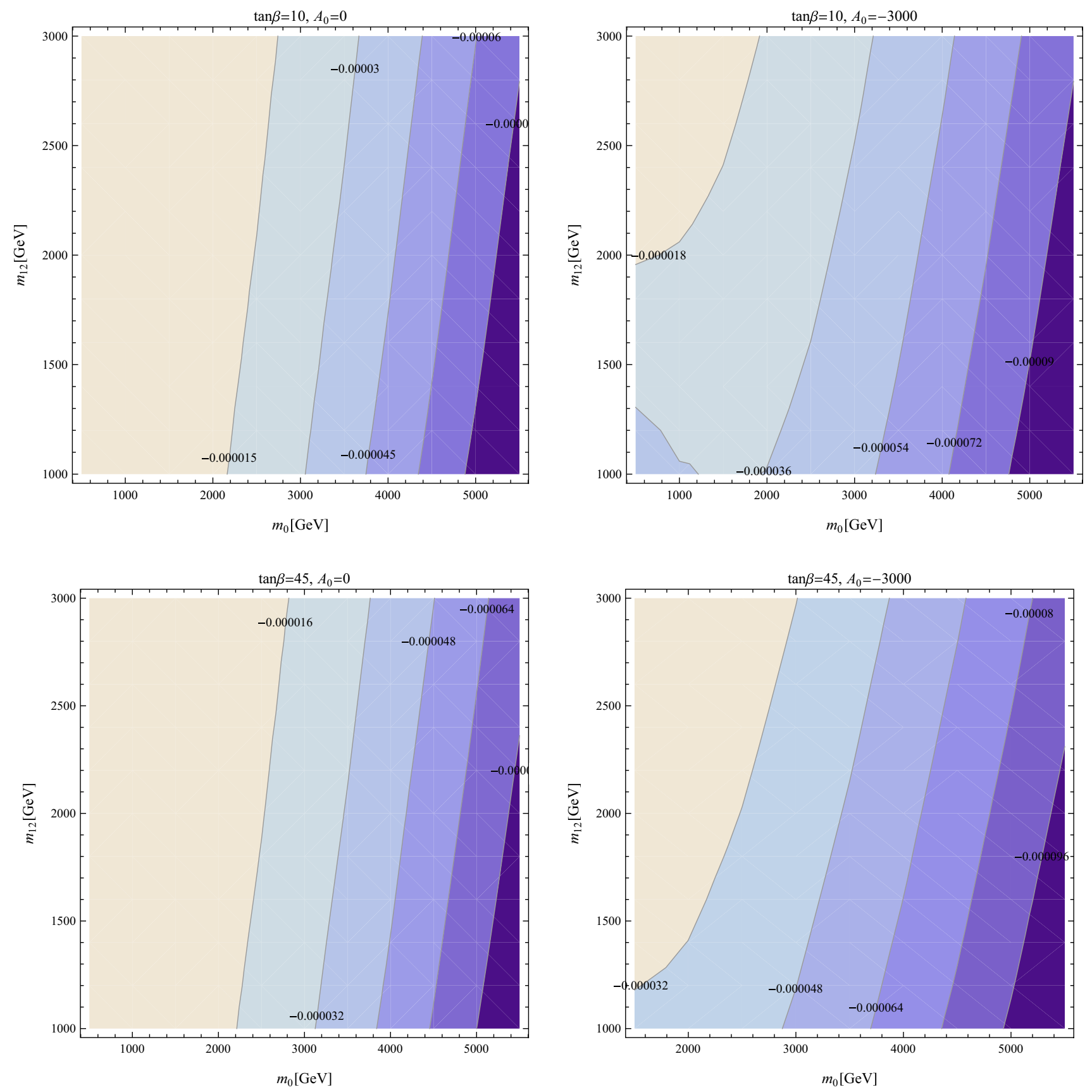

Fig. 14 Contours of $\Delta \sin ^{2} \theta_{\text {eff }}^{\mathrm{MFV}}$ in the $m_{0}-m_{1 / 2}$ plane for different values of $\tan \beta$ and $A_{0}$ in the CMSSM-seesaw I

insufficient approximation, in particular in view of future improved experimental accuracies.

Finally, in Fig. 15 we present the corrections to the Higgs boson masses induced by slepton-flavor violation. Here we only show $\Delta M_{h}^{\mathrm{MFV}}$ (left) and $\Delta M_{H^{ \pm}}^{\mathrm{MFV}}$ (right) for $\tan \beta=10$ and $A_{0}=0$. They turn out to be negligibly small in both cases. Corrections to $\Delta M_{H}^{\mathrm{MFV}}$, which are not shown, are even smaller. We have checked that these results hold also for other combinations of $\tan \beta$ and $A_{0}$. Consequently, within the Higgs sector the approximation of neglecting the effects of the $\delta_{i j}^{F A B}$ is fully justified.

\section{Conclusions}

In this paper we have investigated the CMSSM and the CMSSM-seesaw I (i.e. the CMSSM augmented by righthanded neutrinos to produce the observed neutrino mass pat- 

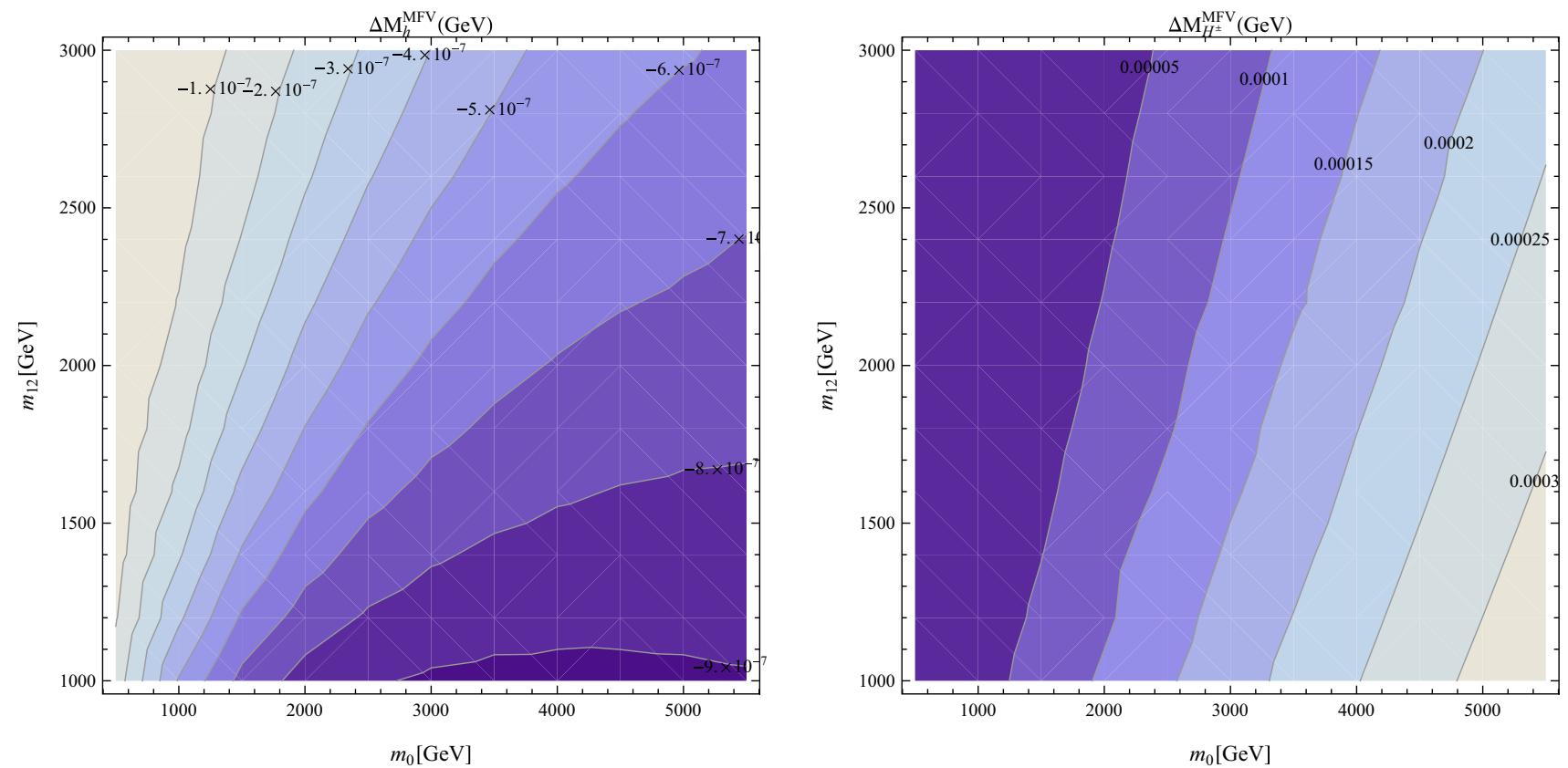

Fig. 15 Contours of $\Delta M_{h}^{\mathrm{MFV}}$ (left) and $\Delta M_{H^{ \pm}}^{\mathrm{MFV}}($ right $)$ in the $m_{0}-m_{1 / 2}$ plane for tan $\beta=10$ and $A_{0}=0$ in the CMSSM-seesaw I

tern via the seesaw type I mechanism) under the hypothesis of Minimal Flavor Violation (MFV, i.e. the only source for flavor violation is the CKM matrix and/or the PMNS matrix in the case of the CMSSM-seesaw I). In many phenomenological analyses of the CMSSM the effects of intergenerational mixing in the squark and/or slepton sector are neglected. However, such mixings are naturally induced, assuming no flavor violation at the GUT scale, by the RGE running from the GUT to the EW scale exactly due to the presence of the CKM and/or the PMNS matrix. In this sense the CMSSM and the CMSSM-seesaw I represent two simple "realistic" GUT based models, in which flavor violation in induced solely by RGE running. The spectra of the CMSSM and CMSSM-seesaw I have been numerically evaluated with the help of the program SPheno by taking the GUT scale input run down via the appropriate RGEs to the EW scale.

We have evaluated the predictions for $B$-physics observables, MSSM Higgs-boson masses, electroweak precision observables in the CMSSM and CMSSM-seesaw I. In order to numerically analyze the effects of neglecting intergenerational mixing these observables have been evaluated with the full spectrum at the EW scale, as well as with the spectrum, but with all intergenerational mixing set numerically (artificially) to zero (as has been done in many phenomenological analyses). We did not attempt an analytical evaluation of the flavor-violation effects, as they become very involved in the case intergenerational sfermion mixing in SUSY models. The numerical difference in the various observables indicates the possible size of the effects neglected in those analyses. In this way it can be checked whether neglecting those mixing effects is a justified approximation.

Within the CMSSM we have taken a fixed grid of $A_{0}$ and $\tan \beta$, while scanning the $m_{0}-m_{1 / 2}$ plane. We found that the value of $\delta_{i j}^{F A B}$ increases with the increase of the $A_{0}$ or $\tan \beta$ values. The Higgs-boson masses receive corrections below current and future experimental uncertainties, where the shifts in $M_{H^{ \pm}}$were found largest at the level of $\mathcal{O}(100 \mathrm{MeV})$. Similarly for the $B$-physics observables the induced effects are at least one order of magnitude smaller than the current experimental uncertainty. For those two groups of observables the approximation of neglecting intergenerational mixing explicitly is a viable option.

The picture changes for the electroweak precision observables. We find that the masses of the squarks grow with $m_{0}$, and so do the mixing terms, inducing a splitting between masses in an $S U$ (2) doublet, leading (numerically) to a nondecoupling effect. For $m_{0} \gtrsim 3 \mathrm{TeV}$ the effects induced in $M_{W}$ and $\sin ^{2} \theta_{\text {eff }}$ are found to be several times larger than the current experimental uncertainties and could shift the CMSSM prediction outside the allowed experimental range. In this way, taking the intergenerational mixing into account could in principle set bounds on $m_{0}$ not present in recent phenomenological analyses. By investigating numerically squark mass differences, we have shown that this behavior can be traced back to the non-decoupling effects in the scalar quark mass matrices, provided by Spheno when taking into account the CKM matrix in the RGE running. However, we would like to point out that this bound only holds because of the particularly simple structure of the CMSSM and cannot 
be extended easily to other, more complicated model frameworks.

Going to the CMSSM-seesaw I the numerical results depend on the concrete model definition. We have chosen a set of parameter that reproduces correctly the observed neutrino data and simultaneously induces large LFV effects and induces relatively large corrections to the calculated observables. Consequently, parts of the parameter space are excluded by the experimental bounds on $\operatorname{BR}(\mu \rightarrow e \gamma)$. Concerning the precision observables we find that $B$-physics observables are not affected, we also find that the additional effects induced by slepton-flavor violation on the Higgsboson masses are negligible. Again the EWPO are found to show the largest impact, where for $M_{W}$ numerical effects at the same level as the current experimental accuracy have been observed for very large values of $m_{0}$. As above, we would like to point out that these effects are due to the relatively simple structure of the CMSSM-seesaw I.

To summarize: we have numerically analyzed two "realistic" GUT based models in which flavor violation in solely induced by the CKM matrix via RGE running (as evaluated using the Spheno code). We find that artificially setting all flavor-violating terms to zero in the CMSSM and CMSSMseesaw $\mathrm{I}$ is an acceptable approximation for $B$-physics observables, Higgs-boson masses (evaluated using a private version of FeynHiggs). However, in the electroweak precision observables (also evaluated with FeynHiggs) in our numerical analysis we find larger effects in the CMSSM and CMSSM-seesaw I. The numerical contributions are larger than the current experimental accuracy in $M_{W}$ and $\sin ^{2} \theta_{\text {eff }}$. Taking those effects correctly into account could in principle place new bounds on $m_{0}$ not present in recent phenomenological analyses.

Acknowledgments The work of S.H. and M.R. was partially supported by CICYT (grant FPA 2013-40715-P). M.G., S.H. and M.R. were supported by the Spanish MICINN's Consolider-Ingenio 2010 Programme under grant MultiDark CSD2009-00064. M.E.G. acknowledges further support from the MICINN project FPA2011-23781.

Open Access This article is distributed under the terms of the Creative Commons Attribution 4.0 International License (http://creativecomm ons.org/licenses/by/4.0/), which permits unrestricted use, distribution, and reproduction in any medium, provided you give appropriate credit to the original author(s) and the source, provide a link to the Creative Commons license, and indicate if changes were made. Funded by SCOAP ${ }^{3}$.

\section{References}

1. H. Nilles, Phys. Rep. 110, 1 (1984)

2. H. Haber, G. Kane, Phys. Rep. 117, 75 (1985)

3. R. Barbieri, Riv. Nuovo Cim. 11, 1 (1988)

4. Y. Amhis et al., [Heavy Flavor Averaging Group], SLAC-R-1002, FERMILAB-PUB-12-871-PPD. arXiv:1207.1158 [hep-ex]
5. R. Chivukula, H. Georgi, Phys. Lett. B 188, 99 (1987)

6. L. Hall, L. Randall, Phys. Rev. Lett. 65, 2939 (1990)

7. A. Buras et al., Phys. Lett. B 500, 161 (2001)

8. G. D'Ambrosio et al., Nucl. Phys. B 645, 155 (2002)

9. S.S. AbdusSalam et al., Eur. Phys. J. C 71, 1835 (2011). arXiv:1109.3859 [hep-ph]

10. B. Allanach et al., Comput. Phys. Commun. 180, 8 (2009). arXiv:0801.0045 [hep-ph]

11. F. Borzumati, A. Masiero, Phys. Rev. Lett. 57, 961 (1986)

12. S. Fukuda et al., [Super-Kamiokande Collaboration], Phys. Rev. Lett. 86, 5656 (2001). arXiv:hep-ex/0103033

13. S. Fukuda et al., [Super-Kamiokande Collaboration], Phys. Rev. Lett. 86, 5651 (2001). arXiv:hep-ex/0103032

14. S. Fukuda et al., [Super-Kamiokande Collaboration], Phys. Lett. B 539, 179 (2002). arXiv:hep-ex/0205075

15. M. Apollonio et al., [CHOOZ Collaboration], Phys. Lett. B 466, 415 (1999). arXiv:hep-ex/9907037

16. Q. Ahmad et al., [SNO Collaboration], Phys. Rev. Lett. 87, 071301 (2001). arXiv:nucl-ex/0106015

17. Q. Ahmad et al., [SNO Collaboration], Phys. Rev. Lett. 89, 011301 (2002). arXiv:nucl-ex/0204008

18. M. Ambrosio et al., [MACRO Collaboration], Phys. Lett. B 517, 59 (2001)

19. G. Giacomelli, M. Giorgini, [MACRO Collaboration], arXiv:hep-ex/0110021

20. K. Eguchi et al., [KamLAND Collaboration], arXiv:hep-ex/0212021

21. V. Cirigliano et al., Nucl. Phys. B 728, 121 (2005). arXiv:hep-ph/0507001

22. P. Minkowski, Phys. Lett. B 67, 421 (1977)

23. M. Gell-Mann, P. Ramond, R. Slansky, in Complex Spinors and Unified Theories, eds. P. Van Nieuwenhuizen, D. Freedman, Supergravity (North-Holland, Amsterdam, 1979), p. 315 [print-80-0576 (CERN)]

24. T. Yanagida, in Proceedings of the Workshop on the Unified Theory and the Baryon Number in the Universe, eds. O. Sawada, A. Sugamoto (KEK, Tsukuba, 1979), p. 95

25. S. Glashow, in Quarks and Leptons, eds. M. Lévy et al. (Plenum Press, New York, 1980), p. 687

26. R. Mohapatra, G. Senjanović, Phys. Rev. Lett. 44, 912 (1980)

27. J. Schechter, J.W.F. Valle, Phys. Rev. D 22, 2227 (1980)

28. J. Schechter, J.W.F. Valle, Phys. Rev. D 25, 774 (1982)

29. O. Buchmueller et al., Eur. Phys. J. C 74, 12, 3212 (2014). arXiv: 1408.4060 [hep-ph]

30. O. Buchmueller et al., Eur. Phys. J. C 74, 2922 (2014). arXiv:1312.5250 [hep-ph]

31. O. Buchmueller et al., Eur. Phys. J. C 72, 2243 (2012). arXiv:1207.7315 [hep-ph]

32. O. Buchmueller et al., Eur. Phys. J. C 72, 1878 (2012). arXiv:1110.3568 [hep-ph]

33. W. Porod, Comput. Phys. Commun. 153, 275 (2003). arXiv:hep-ph/0301101

34. W. Porod, F. Staub, Comput. Phys. Commun. 183, 2458 (2012). arXiv:1104.1573 [hep-ph]

35. P. Skands et al., JHEP 0407, 036 (2004). arXiv:hep-ph/0311123

36. S. Heinemeyer, W. Hollik, G. Weiglein, Comput. Phys. Commun. 124, 76 (2000). arXiv:hep-ph/9812320

37. T. Hahn, S. Heinemeyer, W. Hollik, H. Rzehak, G. Weiglein, Comput. Phys. Commun. 180, 1426 (2009) (see http://www.feynhiggs. de)

38. S. Heinemeyer, W. Hollik, G. Weiglein, Eur. Phys. J. C 9, 343 (1999). arXiv:hep-ph/9812472

39. G. Degrassi, S. Heinemeyer, W. Hollik, P. Slavich, G. Weiglein, Eur. Phys. J. C 28, 133 (2003). arXiv:hep-ph/0212020

40. M. Frank, T. Hahn, S. Heinemeyer, W. Hollik, R. Rzehak, G. Weiglein, JHEP 0702, 047 (2007). arXiv:hep-ph/0611326 
41. T. Hahn, S. Heinemeyer, W. Hollik, H. Rzehak, G. Weiglein, Phys. Rev. Lett. 112, 141801 (2014). arXiv:1312.4937 [hep-ph]

42. G. Isidori, P. Paradisi, Phys. Lett. B 639, 499 (2006). arXiv:hep-ph/0605012

43. G. Isidori, F. Mescia, P. Paradisi, D. Temes, Phys. Rev. D 75, 115019 (2007). arXiv:hep-ph/0703035 (references therein)

44. M. Arana-Catania, S. Heinemeyer, M. Herrero, S. Peñaranda, JHEP 1205, 015 (2012). arXiv:1109.6232 [hep-ph]

45. M. Arana-Catania, S. Heinemeyer, M. Herrero, S. Peñaranda arXiv:1201.6345 [hep-ph]

46. M. Arana-Catania, S. Heinemeyer, M.J. Herrero, Phys. Rev. D 90, 075003 (2014). arXiv:1405.6960 [hep-ph]

47. N. Falck, Z. Phys. C 30, 247 (1986)

48. S. Bertolini, F. Borzumati, A. Masiero, G. Ridolfi, Nucl. Phys. B 353, 591 (1991)

49. J. Hisano, T. Moroi, K. Tobe, M. Yamaguchi, Phys. Rev. D 53, 2442 (1996)

50. M. Cannoni, J. Ellis, M. Gómez, S. Lola, Phys. Rev. D 88(7), 075005 (2013). arXiv:1301.6002[hep-ph]

51. M. Gómez, G. Leontaris, S. Lola, J. Vergados, Phys. Rev. D 59, 116009 (1999). arXiv:hep-ph/9810291

52. J. Ellis, M.E. Gómez, G. Leontaris, S. Lola, D. Nanopoulos, Eur. Phys. J. C 14, 319 (2000). arXiv:hep-ph/9911459

53. S. Antusch, E. Arganda, M. Herrero, A. Teixeira, JHEP 0611, 090 (2006). arXiv:hep-ph/0607263

54. J. Ellis, M. Gómez, S. Lola, JHEP 0707, 052 (2007). arXiv:hep-ph/0612292

55. J. Casas, A. Ibarra, Nucl. Phys. B 618, 171 (2001). arXiv:hep-ph/0103065

56. G. Fogli, E. Lisi, A. Marrone, D. Montanino, A. Palazzo, A. Rotunno, Phys. Rev. D 86, 013012 (2012). arXiv:1205.5254 [hep$\mathrm{ph}]$

57. M. Gómez, T. Hahn, S. Heinemeyer, M. Rehman, Phys. Rev. D 90, 074016 (2014). arXiv:1408.0663 [hep-ph]

58. M. Arana-Catania, S. Heinemeyer, M. Herrero, Phys. Rev. D 88(1), 015026 (2013). arXiv:1304.2783[hep-ph]

59. Y. Kuno, Y. Okada, Rev. Mod. Phys. 73, 151 (2001). arXiv:hep-ph/9909265

60. S. Bilenky, S. Petcov, B. Pontecorvo, Phys. Lett. B 67, 309 (1977)

61. W. Marciano, A. Sanda, Phys. Lett. B 67, 303 (1977)

62. T. Cheng, L.-F. Li, Phys. Rev. Lett. 45, 1908 (1980)

63. A. Djouadi, Phys. Rep. 459, 1 (2008). arXiv:hep-ph/0503173

64. S. Heinemeyer, Int. J. Mod. Phys. A 21, 2659 (2006). arXiv:hep-ph/0407244

65. G. Aad et al., [ATLAS Collaboration], Phys. Rev. D 90, 052004 (2014). arXiv:1406.3827 [hep-ex]
66. CMS Collaboration, [CMS Collaboration], CMS-PAS-HIG-14009

67. H. Baer et al., arXiv:1306.6352 [hep-ph]

68. S. Gennai et al., Eur. Phys. J. C 52, 383 (2007). arXiv:0704.0619 [hep-ph]

69. S. Heinemeyer, W. Hollik, F. Merz, S. Peñaranda, Eur. Phys. J. C 37, 481 (2004). arXiv:hep-ph/0403228

70. K. Kowalska, JHEP 1409, 139 (2014). arXiv:1406.0710 [hep-ph]

71. S. Heinemeyer, W. Hollik, G. Weiglein, Phys. Rep. 425, 265 (2006). arXiv:hep-ph/0412214

72. S. Schael et al., [ALEPH and DELPHI and L3 and OPAL and LEP Electroweak Collaborations], Phys. Rep. 532, 119 (2013). arXiv:1302.3415[hep-ex] (see http://www.cern.ch/LEPEWWG)

73. M. Baak et al., arXiv:1310.6708 [hep-ph]

74. A. Freitas et al., arXiv:1307.3962 [hep-ph]

75. S. Heinemeyer, Talk given at the 8 th FCC-ee Physics Workshop, Paris (2014) (see https://indico.cern.ch/event/337673/session/3/ contribution $/ 41 /$ material/slides)

76. M. Veltman, Nucl. Phys. B 123, 89 (1977)

77. G. Isidori, A. Retico, JHEP 0209, 063 (2002). arXiv:hep-ph/0208159

78. P. Chankowski, L. Slawianowska, Phys. Rev. D 63, 054012 (2001). arXiv:hep-ph/0008046

79. J. Foster, K. Okumura, L. Roszkowski, JHEP 0508, 094 (2005). arXiv:hep-ph/0506146

80. See, https://www.slac.stanford.edu/xorg/hfag/rare. Accessed 16 Sept 2015

81. M. Misiak, Acta Phys. Polon. B 40, 2987 (2009). arXiv:0911.1651 [hep-ph]

82. S. Chatrchyan et al., [CMS Collaboration], Phys. Rev. Lett. 111, 101804 (2013). arXiv:1307.5025 [hep-ex]

83. R. Aaij et al., [LHCb Collaboration], Phys. Rev. Lett. 111, 101805 (2013). arXiv:1307.5024 [hep-ex]

84. A. Buras, J. Girrbach, D. Guadagnoli, G. Isidori, Eur. Phys. J. C 72, 2172 (2012). arXiv:1208.0934 [hep-ph]

85. See, https://www.slac.stanford.edu/xorg/hfag/osc/PDG_2013/. Accessed 16 Sept 2015

86. A. Buras, M. Jamin, P. Weisz, Nucl. Phys. B 347, 491 (1990)

87. E. Golowich, J. Hewett, S. Pakvasa, A. Petrov, G. Yeghiyan, Phys. Rev. D 83, 114017 (2011). arXiv:1102.0009 [hep-ph]

88. S.P. Martin, M.T. Vaughn, Phys. Rev. D 50, 2282 (1994)

89. S.P. Martin, M.T. Vaughn, Phys. Rev. D 78, 039903 (2008). arXiv:hep-ph/9311340

90. J. Cao, G. Eilam, K.I. Hikasa, J. Yang. Phys. Rev. D 74, 031701 (2006). arXiv:hep-ph/0604163

91. J. Adam et al., [MEG Collaboration], arXiv:1303.0754 [hep-ex] 